\title{
Dynamic star formation in the massive DR21 filament
}

\author{
N. Schneider ${ }^{1}$, T. Csengeri ${ }^{1}$, S. Bontemps ${ }^{2}$, F. Motte ${ }^{1}$, R. Simon $^{3}$, P. Hennebelle ${ }^{4}$, C. Federrath ${ }^{5}$, and R. Klessen ${ }^{5,6}$ \\ 1 Laboratoire AIM, CEA/DSM - INSU/CNRS - Université Paris Diderot, IRFU/SAp CEA-Saclay, 91191 Gif-sur-Yvette, France \\ e-mail: nschneid@cea.fr \\ 2 OASU/LAB-UMR5804, CNRS, Université Bordeaux 1, 33270 Floirac, France \\ 3 I. Physikalisches Institut, Universität zu Köln, Zülpicher Straße 77, 50937 Köln, Germany \\ ${ }^{4}$ Laboratoire de radioastronomie, UMR CNRS 8112, École normale supérieure et Observatoire de Paris, 75231 Paris, France \\ 5 Zentrum für Astronomie der Universität Heidelberg, Inst. für Theor. Astrophysik, Albert-Ueberle Str. 2, 69120 Heidelberg, Germany \\ ${ }^{6}$ Kavli Institute for Particle Astrophysics and Cosmology, Stanford University, Menlo Park, CA 94025, USA
}

Received 22 March 2010 / Accepted 24 May 2010

\begin{abstract}
Context. The formation of massive stars is a highly complex process in which it is unclear whether the star-forming gas is in global gravitational collapse or an equilibrium state supported by turbulence and/or magnetic fields. In addition, magnetic fields may play a decisive role in the star-formation process since they influence the efficiency of gas infall onto the protostar.

Aims. By studying one of the most massive and dense star-forming regions in the Galaxy at a distance of less than $3 \mathrm{kpc}$, i.e. the filament containing the well-known sources DR21 and DR21(OH), we attempt to obtain observational evidence to help us to discriminate between these two views.

Methods. We use molecular line data from our ${ }^{13} \mathrm{CO} 1 \rightarrow 0$, CS $2 \rightarrow 1$, and $\mathrm{N}_{2} \mathrm{H}^{+} 1 \rightarrow 0$ survey of the Cygnus X region obtained with the FCRAO and high-angular resolution observations in isotopomeric lines of $\mathrm{CO}, \mathrm{CS}, \mathrm{HCO}^{+}, \mathrm{N}_{2} \mathrm{H}^{+}$, and $\mathrm{H}_{2} \mathrm{CO}$, obtained with the IRAM $30 \mathrm{~m}$ telescope, to investigate the distribution of the different phases of molecular gas. Gravitational infall is identified by the presence of inverse P Cygni profiles that are detected in optically thick lines, while the optically thinner isotopomers are found to reach a peak in the self-absorption gap.

Results. We observe a complex velocity field and velocity dispersion in the DR21 filament in which regions of the highest columndensity, i.e., dense cores, have a lower velocity dispersion than the surrounding gas and velocity gradients that are not (only) due to rotation. Infall signatures in optically thick line profiles of $\mathrm{HCO}^{+}$and ${ }^{12} \mathrm{CO}$ are observed along and across the whole DR21 filament. By modelling the observed spectra, we obtain a typical infall speed of $\sim 0.6 \mathrm{~km} \mathrm{~s}^{-1}$ and mass accretion rates of the order of a few $10^{-3} M_{\odot} \mathrm{yr}^{-1}$ for the two main clumps constituting the filament. These massive clumps (4900 and $3300 M_{\odot}$ at densities of around $10^{5} \mathrm{~cm}^{-3}$ within $1 \mathrm{pc}$ diameter) are both gravitationally contracting (with free-fall times much shorter than sound crossing times and low virial parameter $\alpha$ ). The more massive of the clumps, DR21(OH), is connected to a sub-filament, apparently "falling" onto the clump. This filament runs parallel to the magnetic field.

Conclusions. All observed kinematic features in the DR21 filament (velocity field, velocity dispersion, and infall), its filamentary morphology, and the existence of (a) sub-filament(s) can be explained if the DR21 filament was formed by the convergence of flows on large scales and is now in a state of global gravitational collapse. Whether this convergence of flows originated from self-gravity on larger scales or from other processes cannot be determined by the present study. The observed velocity field and velocity dispersion are consistent with results from (magneto)-hydrodynamic simulations where the cores lie at the stagnation points of convergent turbulent flows.
\end{abstract}

Key words. ISM: clouds - ISM: individual objects: Cygnus X - ISM: molecules - ISM: kinematics and dynamics radio lines: ISM - submillimeter: ISM

\section{Introduction}

Our goal in this series of papers is to investigate the generic link between the large-scale (several $10 \mathrm{pc}$ ) molecular-cloud spatial structure (Schneider et al. 2010), medium-scale $(<10 \mathrm{pc})$ dynamic fragment properties (this paper), and the occurrence of high-mass star formation on small $(0.1-0.5 \mathrm{pc})$ scales (Bontemps et al. 2010; Csengeri et al. 2010). To achieve this, we make use of our multiwavelength study in Cygnus X (see below). This region has already been shown to represent an excellent laboratory for studies of high-mass star formation.

Molecular clouds (MCs) are the birthplaces of low- and highmass stars. Understanding their formation and evolution is essential to understanding in general the star formation (SF) process. There are currently two different scenarios for the evolution of MCs and stars, a dynamic and a quasi-static view.
In the dynamic context, MCs are transient, rapidly evolving entities that are not in equilibrium, and the spatial and velocity structure of the cloud is determined by compressible supersonic turbulence (see e.g., Mac Low \& Klessen 2004, for an overview). The driving sources of turbulence can be diverse and their relative importance remains a subject of debate. Large-scale mechanisms such as supernovae explosions, should be the most important, based on theoretical arguments extensively discussed by MacLow \& Klessen (2004).

Turbulence may also occur during the formation process of molecular clouds (Vazquez-Semandeni et al. 2002; Klessen \& Hennebelle 2010) within large-scale colliding flows of mostly atomic gas in the galactic disk, generated by dynamic compression in the interstellar medium or other large-scale instabilities (see, e.g., Hennebelle \& Audit 2007; Vazquez-Semadeni et al. 2008; Hennebelle et al. 2008; Banerjee et al. 2009). High 
velocity compressive flows form dense structures at stagnation points that may collapse to form stars/clusters (BallesterosParedes et al. 2003; Vazquez-Semadeni et al. 2007, 2009; Heitsch et al. 2008). In this case, the velocity field and velocity dispersion of molecular clumps may contain the signature of the external convergent motion and the compressive, gravitational contraction (this was already proposed by Goldreich \& Kwan 1974). The star-forming core is then the dense post-shock region with a more quiescent velocity dispersion than the turbulent flow (Klessen et al. 2005). This scenario is valid for both, low- and high-mass star formation. However, there is no direct observational confirmation of the existence of convergent flows. Studying the dynamics of $\mathrm{HI}$ and molecular gas may be a possibility (Brunt 2003), though it is not clear what observational signatures are to be expected. An indirect argument for molecular cloud formation out of colliding HI flows was provided by Audit \& Hennebelle (2010), who showed that their models closely reproduce the observed clump mass spectra and Larson-relations. It has also been noted that the lifetime of the cloud in the gravoturbulent framework is short (one dynamical crossing time, i.e. $\sim 10^{7}$ yr for giant molecular clouds) and the star formation process is rapid.

In the quasi-static view (see McKee \& Ostriker 2007, and references therein), MCs are formed by large-scale selfgravitating instabilities such as spiral density waves. "Highmass" star formation is approached in the "turbulent core" model (McKee \& Tan 2003) in which the star-forming cores are supersonically turbulent. Turbulent magnetic and thermal pressure supports the clump against self-gravity. Because of energy injection from newly formed stars, the clumps and most of the cores may maintain their equilibrium, unless they quasistatically evolve to a gravitationally unstable state to finally form stars (if the magnetic flux diffuses out of the clump by ambipolar diffusion). In the case of low-mass stars, the collapse of a rotating cloud of gas and dust leads to the formation of an accretion disk through which matter is channeled onto a central protostar. For stars with masses higher than about $8 M_{\odot}$ this mechanism of star formation is less clear due to the strong radiation field that pushes against infalling material and may halt accretion. However, theoretical work (Yorke \& Sonnhalter 2002; Krumholz et al. 2005; Peters et al. 2010) has shown that outflows lead to anisotropy in the stellar radiation field, which reduce the radiation effects experienced by gas in the infalling envelope. Thus, massive stars may therefore be able to form by a mechanism similar to that by which low mass stars form. Peters et al. (2010) even show that clustered SF is a natural outcome of massive SF even in the presence of radiative feedback. However, clustered $\mathrm{SF}$ is also a natural result of the competitive accretion scenario (Bonnell \& Bate 2006), in which the fragmentation of a turbulent cloud produces stars with masses of the order of the Jeans mass within a common gravitational potential. These stars, located close to the centre of the potential, accrete at much higher rates than isolated stars and become massive.

Both scenarios have in common that massive $\left(>10 M_{\odot}\right)$ stars form only from the coldest, and densest, cores (size scale $<0.1 \mathrm{pc}$ ) of molecular clouds and that high infall/accretion rates are required to overcome feedback processes (ionizing radiation, jets, outflows). In the dynamic framework, stars form as the dynamical evolution of the MC progresses with gas continuously being accreted. Individual clumps $(\sim 0.1-0.5 \mathrm{pc})$ fragment into hundreds of protostars, possibly competing for mass in the central regions of the cluster (Bonnell \& Bate 2006). Support from magnetic fields (Hennebelle \& Teyssier 2008) and/or radiation (Krumholz 2006, Bate 2009) could drastically limit the level of fragmentation and channel the global infall to fewer, more massive protostars (Bontemps et al. 2010).

From the observational point of view, indications of global collapse have been detected using molecular lines. Spectral profiles of high density tracers, usually combining at least an optically thin and an optically thick line, are good probes of infalling gas (e.g. Myers et al. 1996). Observations in low-mass star-forming regions (e.g. Lee et al. 2003) contain a mixture of infall (blue-shifted emission and/or redshifted self-absorption in the optically thick line) and outflow asymmetry. This sort of line profile was also found in the high-mass star-forming region W43 (Motte et al. 2003). Peretto et al. (2006) proposed that a peculiar velocity discontinuity could be the result of some large-scale motion, originating from self-gravity (Peretto et al. 2007). The theory of turbulent core formation, on the other hand, predicts the existence of massive pre-stellar cores that have not been detected so far, not even in sensitive and extensive dust continuum studies (e.g. Motte et al. 2007).

In this paper, we shortly introduce the Cygnus $\mathrm{X}$ region and the DR21 filament in Sect. 2 and describe the molecular line oberservations in Sect. 3 and show maps and spectra in Sect. 4. Section 5 presents an analysis of the kinematic structure and the physical properties of the DR21 filament and in Sect. 6 we use our findings to test the conditions of the different high-mass star formation and turbulence models. Section 7 summarizes the paper.

\section{Cygnus $X$}

\section{The Cygnus $X$ region}

Cygnus $X$ is one of the richest star-formation regions in the Galaxy, covering an area of about 30 square degrees in the Galactic plane around Galactic longitude $80^{\circ}$ (Reipurth \& Schneider 2008). It contains a prominent OB-association, Cyg OB2, with $\sim 100 \mathrm{O}$-stars and a total stellar mass of up to $10^{5} \mathrm{M}_{\odot}$ (Knödlseder 2000). From large-scale ${ }^{13} \mathrm{CO} 2 \rightarrow 1$ (KOSMA $^{1}$, Schneider et al. 2006) and ${ }^{13} \mathrm{CO} 1 \rightarrow 0\left(\mathrm{FCRAO}^{2}\right.$, Schneider et al. 2007, Simon et al., in prep.) surveys, we obtained a mass of a few $10^{6} M_{\odot}$ for the whole molecular cloud complex that is divided into two parts - Cygnus North and South. These studies also showed that the majority of molecular clouds is located at a common distance of about $1.7 \mathrm{kpc}$, i.e. the distance of Cyg OB2.

Its proximity makes Cygnus $\mathrm{X}$ one of the rare laboratories where different phases of massive star formation can be studied in detail. More than 800 distinct HII regions, a number of Wolf-Rayet stars, several OB associations, and at least one star of spectral type O4If are known in Cygnus X, reflecting its record of high-mass star formation in the past. Ongoing massive star formation is revealed by wide-field $\left(3 \mathrm{deg}^{2}\right)$ $1.2 \mathrm{~mm}$ continuum imaging of the densest regions in the Cygnus X molecular clouds (Motte et al. 2007). More than 100 protostellar dense-cores were detected of which 40 are likely to be the precursors of massive OB stars. High-angular resolution observations with the Plateau de Bure Interferometer (Bontemps et al. 2010) have indeed confirmed that the most massive dense cores of this sample are the sites of massive star formation, mainly in the form of clusters.

\section{The DR21 filament}

From our $1.2 \mathrm{~mm}$ continuum imaging and the ${ }^{13} \mathrm{CO}$ surveys, we concluded that the molecular ridge containing DR21 and

\footnotetext{
${ }^{1}$ Cologne Observatory for Submm-Astronomy.

${ }^{2}$ Five College Radio Astronomy Observatory.
} 
DR21(OH) (Dickel et al. 1978; Wilson \& Mauersberger 1990; Jakob et al. 2007) is the most active, dense (average density $\sim 10^{4} \mathrm{~cm}^{-3}$ ), and massive (34000 $M_{\odot}$ ) cloud in Cygnus X.

DR21 (Downes \& Rinehart 1966) itself is a group of several compact HII regions (Harris et al. 1973) with an associated, very energetic outflow (e.g. Garden et al. 1991a; Russell et al. 1992), located in the southern part of the ridge. Three arcminutes further north lies DR21(OH), famous for its maser emission $\left(\mathrm{H}_{2} \mathrm{O}\right.$, Genzel \& Downes 1977; OH, Norris et al. 1982; $\mathrm{CH}_{3} \mathrm{OH}$, Batrla \& Menten 1988). Even further north lies the massive star-forming region W75N (see Shepherd et al. 2004, for a review). Very recently, the DR21 filament was again the target for a wealth of studies in various wavelengths. Infrared images at 3.6, 4.5, and $8 \mu \mathrm{m}$ from the Spitzer satellite (Marston et al. 2004; Hora et al. 2009) show the complexity of the region with many IR-filaments perpendicular to the ridge that seem to be sites of star formation (Kumar et al. 2007). Dust continuum studies (Vallée \& Fiege 2006; Motte et al. 2007) confirmed the detection of 8 compact, dense cores in the DR21 filament (Chandler et al. 1993) and found additional ones. Some of them are high mass protostars and drive outflows detected in $\mathrm{SiO}$ (Motte et al. 2007). An $\mathrm{H}_{2}$ 1-0 S(1) image at $2.121 \mu \mathrm{m}$ (Davis et al. 2007) shows at least 50 individual outflows driven by embedded lowmass stars.

We studied this particular filament in high-angular resolution observations with the IRAM ${ }^{3} 30 \mathrm{~m}^{2}$ telescope in different molecular tracers (isotopomeric $\mathrm{CO}$ lines, $\mathrm{CS}, \mathrm{C}^{34} \mathrm{~S}, \mathrm{HCO}^{+}$, $\mathrm{H}^{13} \mathrm{CO}^{+}, \mathrm{N}_{2} \mathrm{H}^{+}, \mathrm{H}_{2} \mathrm{CO}$ ) to investigate the distribution of the different phases of molecular gas (cold dense cores, warm envelopes) and to uncover infall/outflow signatures. The lower angular resolution FCRAO data delineate the large-scale structure in which the DR21 filament is embedded. A more detailed analysis of the FCRAO ${ }^{13} \mathrm{CO}$ data set is presented in Schneider et al. (2007, 2010) and the CS and $\mathrm{N}_{2} \mathrm{H}^{+}$data will be discussed in a forthcoming paper. All molecular line data we obtained serve as a basis for a physical model of the DR21 filament, including detailed non-LTE line modelling.

\section{Observations}

\subsection{FCRAO}

We used data from a molecular line survey $\left({ }^{13} \mathrm{CO}\right.$ and $\mathrm{C}^{18} \mathrm{O}$ $J=1 \rightarrow 0$, CS $J=2 \rightarrow 1$, and $\mathrm{N}_{2} \mathrm{H}^{+} J=1 \rightarrow 0$ ) of the entire Cygnus X region (Simon et al., in prep.; Schneider et al. 2007) taken with the FCRAO $14 \mathrm{~m}$ telescope. The data were obtained between 2003 December and 2006 January using the 32 pixel "second quabbin optical imaging array" (SEQUOIA) in an On-the-Fly (OTF) observing mode. In this paper, we employ ${ }^{13} \mathrm{CO} 1 \rightarrow 0, \mathrm{CS} 2 \rightarrow 1$, and $\mathrm{N}_{2} \mathrm{H}^{+} 1 \rightarrow 0$ data that cover an area of 35 square degrees in ${ }^{13} \mathrm{CO}$ and 12 square degrees in $\mathrm{CS} / \mathrm{N}_{2} \mathrm{H}^{+}$ on a $22^{\prime \prime} .5$ grid. The beamwidth of the FCRAO at 93 (110) $\mathrm{GHz}$ is $45^{\prime \prime}\left(48^{\prime \prime}\right)$ and the dual channel correlator was configured to obtain a velocity resolution of $\sim 0.1 \mathrm{~km} \mathrm{~s}^{-1}$. The spectra have a mean $1 \sigma \mathrm{rms}$ noise level of $\sim 0.2 \mathrm{~K}$ on a $T_{\mathrm{A}}^{*}$ antenna temperature scale, i.e., not corrected for the main beam efficiency of $\sim 0.48$. Pointing and calibration were checked regularly at the start of the Cygnus observing interval and after transit (no observations were performed at elevations higher than $75^{\circ}$ ). Pointing sources were $\mathrm{SiO}$ masers of evolved stars, i.e., $\chi$-Cyg, R-Leo and T-Cep, depending on LST-time. The calibration was checked regularly on the position of peak emission in DR21 and found to be consistent to within $10 \%$.

3 Institut de Radio-Astronomie Millimétrique.
Table 1. Observing parameters of the molecular line data obtained with the FCRAO and IRAM $30 \mathrm{~m}$ telescope.

\begin{tabular}{lcccccc}
\hline \hline & $\begin{array}{c}v \\
{[\mathrm{GHz}]}\end{array}$ & $H P B W$ & $\eta_{\mathrm{mb}}$ & $\begin{array}{c}T_{\text {sys }} \\
{[\mathrm{K}]}\end{array}$ & $\begin{array}{c}\Delta v_{\text {res }} \\
{\left[\mathrm{km} \mathrm{s}^{-1}\right]}\end{array}$ & $\begin{array}{c}\Delta T_{\mathrm{rms}} \\
{[\mathrm{K}]}\end{array}$ \\
\hline FCRAO & & & & & & \\
${ }^{13} \mathrm{CO} 1 \rightarrow 0$ & 110.2 & $45^{\prime \prime}$ & 0.48 & 210 & 0.067 & 0.48 \\
$\mathrm{CS} 2 \rightarrow 1$ & 98.0 & $48^{\prime \prime}$ & 0.48 & 200 & 0.075 & 0.38 \\
$\mathrm{~N}_{2} \mathrm{H}^{+} 1 \rightarrow 0$ & 93.2 & $48^{\prime \prime}$ & 0.48 & 200 & 0.079 & 0.38 \\
\hline $\mathrm{IRAM}$ & & & & & & \\
${ }^{12} \mathrm{CO} 2 \rightarrow 1$ & 230.8 & $11^{\prime \prime}$ & 0.52 & 601 & 0.05 & 0.8 \\
$\mathrm{H}_{2} \mathrm{CO} 3(1,2)-2(1,1)$ & 225.7 & $11^{\prime \prime}$ & 0.55 & 549 & 0.05 & 0.65 \\
${ }^{13} \mathrm{CO} 2 \rightarrow 1$ & 220.4 & $11^{\prime \prime}$ & 0.57 & 580 & 0.03 & 0.9 \\
$\mathrm{C}^{34} \mathrm{~S} 2 \rightarrow 1$ & 96.4 & $26^{\prime \prime}$ & 0.77 & 210 & 0.025 & 0.5 \\
$\mathrm{~N}_{2} \mathrm{H}^{+} 1 \rightarrow 0$ & 93.2 & $26^{\prime \prime}$ & 0.77 & 158 & 0.03 & 0.1 \\
$\mathrm{HCO}^{+} 1 \rightarrow 0$ & 89.2 & $28^{\prime \prime}$ & 0.77 & 138 & 0.05 & 0.2 \\
$\mathrm{H}^{13} \mathrm{CO}^{+} 1 \rightarrow 0$ & 86.8 & $29^{\prime \prime}$ & 0.78 & 114 & 0.025 & 0.25 \\
\hline
\end{tabular}

Notes. Column one and two indicate the molecular transition and frequency, followed by the half power beam width $(H P B W)$ in $\operatorname{arcsec}, \eta_{\mathrm{mb}}$ is the main beam efficiency, $T_{\text {sys }}$ the system temperature, $\Delta v_{\text {res }}$ denotes the velocity resolution, and $\Delta T_{\text {rms }}$ the average rms noise temperature per channel on a $T_{\mathrm{mb}}$ scale.

\subsection{IRAM}

All molecular line maps ${ }^{4}$ obtained with the IRAM 30 m telescope in the Sierra Nevada/Spain were performed in June 2007. Table 1 gives an overview of all lines and observational parameters. In order to efficiently map an area of $\sim 4^{\prime} \times 10^{\prime}$, covering the whole DR21 filament, we observed in the "on-the-fly" mapping mode. The total area was divided into 7 sub-maps that were scanned horizontally. For strong lines (e.g. $\mathrm{CO}, \mathrm{HCO}^{+}$), only one or two coverages were required while weaker lines were observed more often or with two receivers at once to obtain a sufficient signal-to-noise ratio. The resulting regridded $\left(5^{\prime \prime}\right)$ maps show slight smearing effects and fluctuations in the intensity calibration only in the lines of ${ }^{12} \mathrm{CO}$ and $\mathrm{H}_{2} \mathrm{CO}$. The center position of all maps is within $3^{\prime \prime}$ of the MM2-position given in Mangum et al. (1991): $\operatorname{RA}(2000)=20^{\mathrm{h}} 39^{\mathrm{m}} 00^{\mathrm{s}}$ and $\operatorname{Dec}(2000)=42^{\circ} 22^{\prime} 44^{\prime \prime}$. As OFF-position, we used a position with offset $0^{\prime \prime}, 1800^{\prime \prime}$ from DR21(OH) where ${ }^{12} \mathrm{CO} 2 \rightarrow 1$ emission of around $1 \mathrm{~K}$ is observed at velocities around $-42 \mathrm{~km} \mathrm{~s}^{-1}$, separated well from the bulk emission of the DR21 filament. All data were reduced using GILDAS ${ }^{5}$.

The spectral lines were recorded simultaneously in uniform, good weather conditions (average atmospheric opacity of 0.2 at $230 \mathrm{GHz}$ ) prevailing over several days. We used the IRAM facility receivers and autocorrelator, which were adapted in terms of spectral resolution and bandwidth such that all lines have a similar velocity resolution between 0.03 and $0.05 \mathrm{~km} \mathrm{~s}^{-1}$. Pointing and focus were checked every $2 \mathrm{~h}$. The pointing accuracy was found to be better than $4^{\prime \prime}$ and the receivers were aligned to within $2^{\prime \prime}$.

\section{Results}

\subsection{The DR21 filament as part of a complex network}

Figure 1 shows the zeroth moment map of line integrated ( -10 to $20 \mathrm{~km} \mathrm{~s}^{-1}$ ) CS $2 \rightarrow 1$ emission obtained with the FCRAO overlaid on a grey scale map of ${ }^{13} \mathrm{CO} 1 \rightarrow 0$ emission. The moment maps were constructed following the masked moment procedure

\footnotetext{
4 The IRAM data are available on request, contact nschneid@cea.fr.

${ }^{5}$ http:iram.fr/IRAMFR/GILDAS
} 


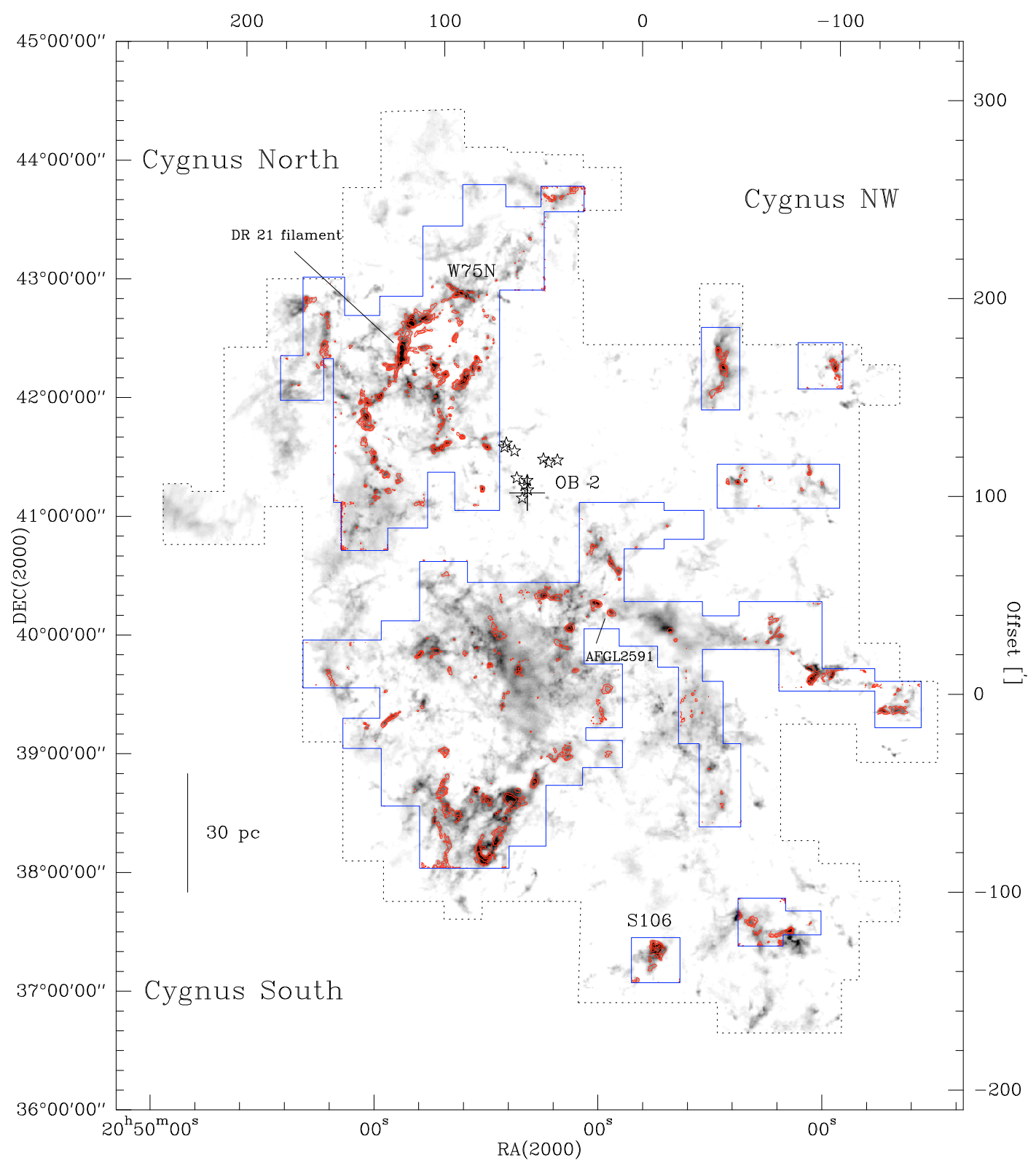

Fig. 1. Zeroth moment map of CS $2 \rightarrow 1$ emission (red contours, mapping area indicated by a continuous blue line) in Cygnus X overlaid on a moment map of ${ }^{13} \mathrm{CO} 1 \rightarrow 0$ emission (mapping area indicated by a dashed line). Both maps were obtained with the FCRAO and have an angular resolution of $\sim 50^{\prime \prime}$. Emission was integrated in the velocity range -10 to $20 \mathrm{~km} \mathrm{~s}^{-1}$ and the grey scaling ranges from 1 to $15 \mathrm{~K} \mathrm{~km} \mathrm{~s}^{-1}$. Red contour levels for CS are at $0.5,1,2,4 \mathrm{~K} \mathrm{~km} \mathrm{~s}^{-1}$. The DR21 filament is indicated, as well as some prominent objects in Cygnus X (S106, AFGL2591, $\mathrm{W} 75 \mathrm{~N}$, the most massive stars of the OB2 cluster).

described in Adler et al. (1992). For CS and $\mathrm{N}_{2} \mathrm{H}^{+}$, we used a temperature threshold of $0.1 \mathrm{~K}$, for ${ }^{13} \mathrm{CO}$ a threshold of $0.2 \mathrm{~K}$. For the CS mapping, we focussed on the high column density regions seen in ${ }^{13} \mathrm{CO}$. We adopt the nomenclature "Cygnus- $\mathrm{X}$ North, South, and Northwest", established in Schneider et al. (2006) for the different cloud regions.

The CS emission is more compact than that of ${ }^{13} \mathrm{CO}$ and clearly delineates the high density regions. Interestingly, regions of peak emission in ${ }^{13} \mathrm{CO}$ do not always show up in CS. This indicates that, though the column density is high, the average density in these regions may stay below the critical density $\left(n_{\mathrm{cr}}\right)$ of the CS line (a few $10^{5} \mathrm{~cm}^{-3}$ ). Abundance variations caused by chemical effects probably also play a role. While in Cygnus North, and partly in its north-west region, CS clumps form a connected network of filamenatry structures, the emission distribution in Cygnus South is more dispersed with more isolated CS clumps.

In the following, we concentrate on a discussion of the filaments in Cygnus-North. For that, we display in Fig. 2 maps of CS $2 \rightarrow 1$ and $\mathrm{N}_{2} \mathrm{H}^{+} 1 \rightarrow 0$ emission where the "organization" of clumps along filaments is clearly visible. Even the $\mathrm{N}_{2} \mathrm{H}^{+}$map - which traces only dense $\left(n_{\mathrm{cr}}=0.5-70 \times 10^{6} \mathrm{~cm}^{-3}\right)$ and cold gas (e.g. Tafalla et al. 2002) - shows the same arrangement of clumps. Most prominent in all maps is the filament containing the HII region DR21 (see below). The moment maps (Fig. 2) show that all evolved star formation sites in Cygnus North (indicated by the HII regions DR17 to DR23) as well as future star-forming sites (indicated by the mm-continuum sources tracing massive dense cores, Motte et al. 2007) lie on filamentary structures. 

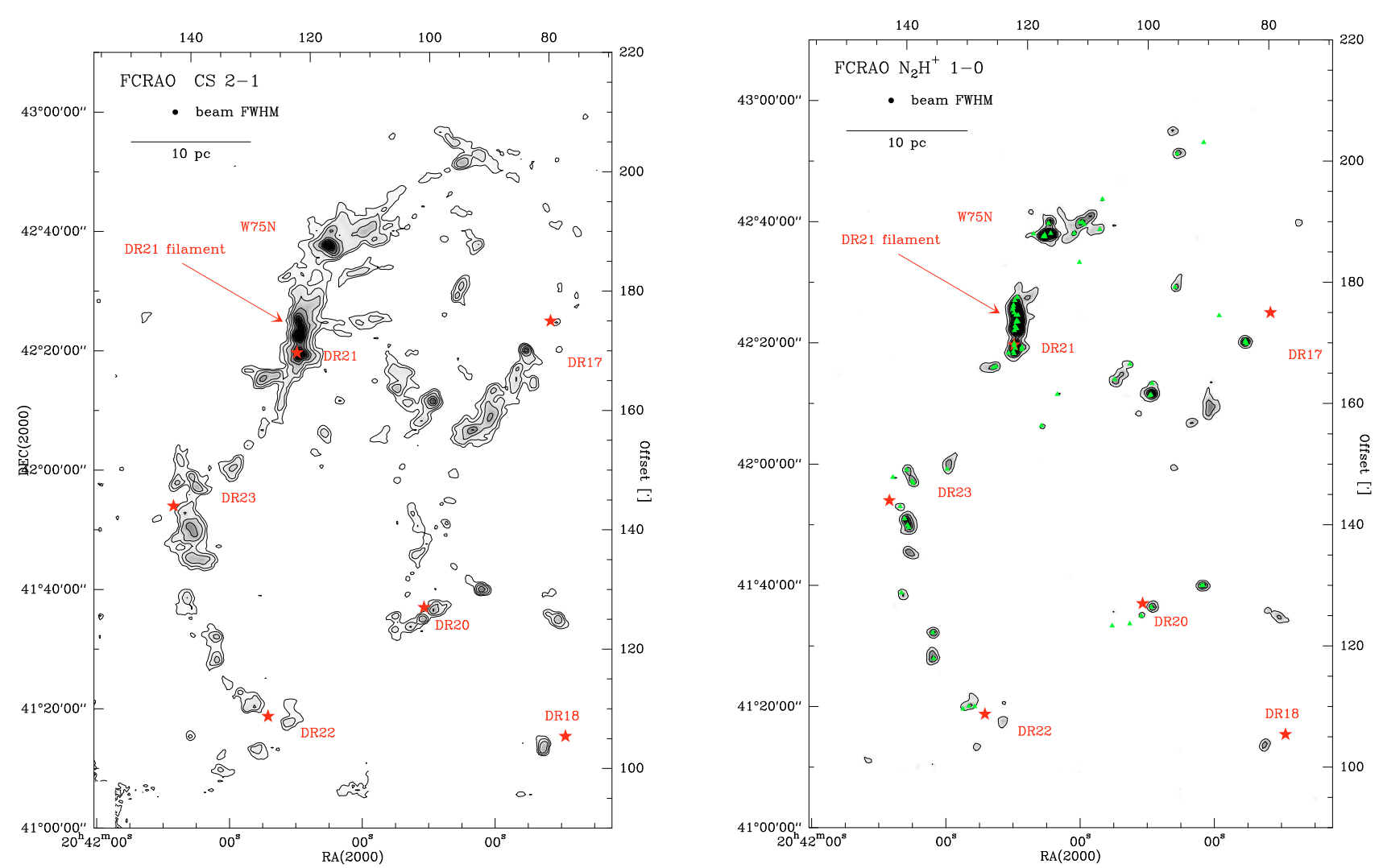

Fig. 2. Zeroth moment maps of CS $2 \rightarrow 1$ (left, contour levels are $0.5,1,2,4 \mathrm{~K} \mathrm{~km} \mathrm{~s}^{-1}$ ) and $\mathrm{N}_{2} \mathrm{H}^{+} 1 \rightarrow 0$ (right, contour levels are 0.5 , 1.5 , $3 \mathrm{~K} \mathrm{~km} \mathrm{~s}^{-1}$ ) emission in Cygnus X North in the velocity range -10 to $20 \mathrm{~km} \mathrm{~s}^{-1}$ observed with the FCRAO. Thermal HII regions (DR17-23) are indicated by red stars, mm-continuum sources from Motte et al. (2007) by green triangles. The latter correspond very well with peaks of $\mathrm{N}_{2} \mathrm{H}^{+}$ emission.

\subsection{The DR21 filament}

From the large-scale, medium-angular-resolution $\left(\sim 1^{\prime}\right)$ molecular line maps (Sect. 4.1) and previous studies (Sect. 1), the DR21 filament clearly appears to be the most prominent starforming region in Cygnus X. The DR21 region in the south contains a very powerful, evolved outflow, while further north, the DR21 $(\mathrm{OH})$ region contains maser sources, indicative of recent star-formation activities. The mm-contiuum survey of Motte et al. (2007) revealed more than 20 protostellar objects, i.e. sites of star formation. The filament is shown in more detail in channel maps of ${ }^{13} \mathrm{CO} 1 \rightarrow 0$ emission (Fig. 3), obtained with the FCRAO. It reveals the characteristic N-S oriented ridge known from other molecular line and continuum surveys (Dickel et al. 1978; Wilson \& Mauersberger 1990; Vallée \& Fiege 2006; Motte et al. 2007; Jakob et al. 2007). What is special in these channel maps is the appearance of several E-W oriented subfilaments (indicated as F1-F3 at $v=-2.6 \mathrm{~km} \mathrm{~s}^{-1}$ ) that are connected to the main DR21 filament and extend over several velocity channels. The most prominent one (F3) has a length of around $10^{\prime}(5 \mathrm{pc})$ and is also visible in the CS map (Fig. 2) and in mm-continuum (Motte et al. 2007), indicating that gas of high density is present.

\subsubsection{Average spectra}

To study in more detail the physical properties (mass, density, temperature) and kinematics (infall and outflow signatures, velocity gradient) of the filament, we mapped the central part of the filament containing DR21 and DR21(OH) in various molecular line tracers at an angular resolution of between $12^{\prime \prime}$ and $30^{\prime \prime}$ using the IRAM $30 \mathrm{~m}$ telescope.

To distinguish the different emission features of the filament, we produced positionally averaged spectra of five of the observed lines (Fig. 4). The averaging was performed across each map extent, i.e. covering the whole filament. The $\mathrm{CO}$ lines exhibit two velocity components, one centered on $-3 \mathrm{~km} \mathrm{~s}^{-1}$ and one on $+9 \mathrm{~km} \mathrm{~s}^{-1}$. They correspond to the molecular clouds associated with DR21 and W75N, respectively (see also Dickel et al. 1978). Though the $\mathrm{W} 75 \mathrm{~N}$ region itself is not included in the mapping region, molecular gas related to this star-forming region northwest of the DR21 filament (see Fig. 2) is still present across the filament. However, the gas is more diffuse and of lower density since a line at $+9 \mathrm{~km} \mathrm{~s}^{-1}$ (Fig. 4) can only be seen in the low-density tracers $\left({ }^{12} \mathrm{CO}\right.$ and $\left.{ }^{13} \mathrm{CO}\right)$. The $\mathrm{HCO}^{+}$line reveals a narrow absorption feature at $+9 \mathrm{~km} \mathrm{~s}^{-1}$ (seen also by Nyman 1983), while the optically thin lines show no significant emission for the $\mathrm{W} 75 \mathrm{~N}$ component.

The bulk emission of the DR21 filament is found at $3 \mathrm{~km} \mathrm{~s}^{-1}$ (single Gaussian lines in $\mathrm{C}^{34} \mathrm{~S}, \mathrm{H}^{13} \mathrm{CO}^{+}$, and ${ }^{13} \mathrm{CO}$ ). The optically thick lines of ${ }^{12} \mathrm{CO}$ and $\mathrm{HCO}^{+}$show prominent, extended blue and red wing emission. The red wing, however, is blended with the $\mathrm{W} 75 \mathrm{~N}$ component, seen in emission in ${ }^{12} \mathrm{CO}$ and absorption in $\mathrm{HCO}^{+}$. The wings are caused by the powerful outflow of DR21 (Garden et al. 1991a) but also other outflow sources discussed in Sect. 4.3. The self-absorption feature at $3 \mathrm{~km} \mathrm{~s}^{-1}$ is seen in the optically thick lines of ${ }^{12} \mathrm{CO}$ and $\mathrm{HCO}^{+}$, while optically thin lines peak at the velocity of the absorption dip. This may be a signature of inflowing gas and is discussed in more detail in Sect. 5.2. 


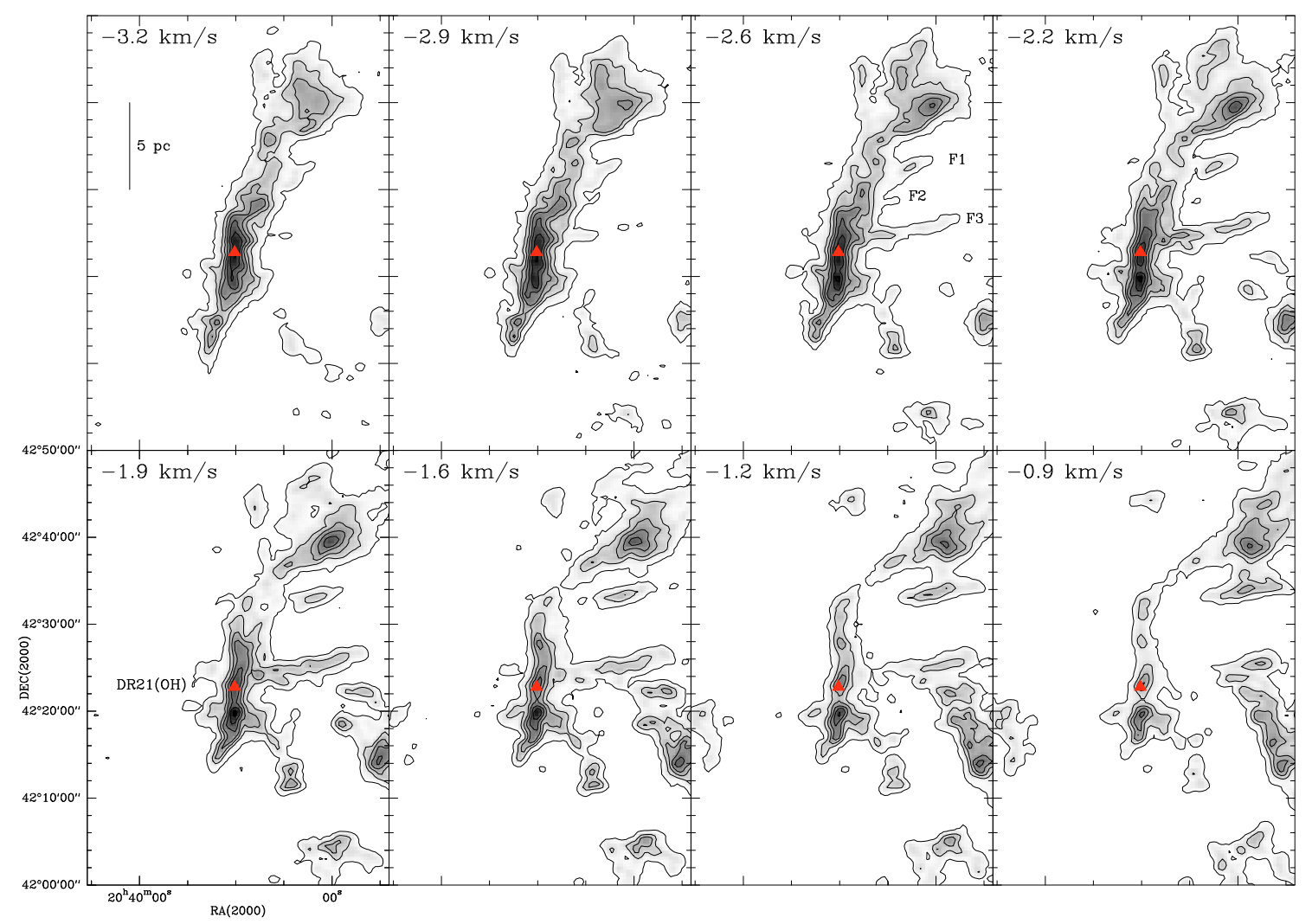

Fig. 3. Channel maps of ${ }^{13} \mathrm{CO} 1 \rightarrow 0$ emission in Cygnus X North in the velocity range -3.2 to $-0.9 \mathrm{~km} \mathrm{~s}^{-1}$ observed with the FCRAO. The red triangle shows the position of DR21 (OH). The three major subfilaments (F1, F2, F3) linked with the large DR21 filament are indicated in the plot of velocity $-2.6 \mathrm{~km} \mathrm{~s}^{-1}$.

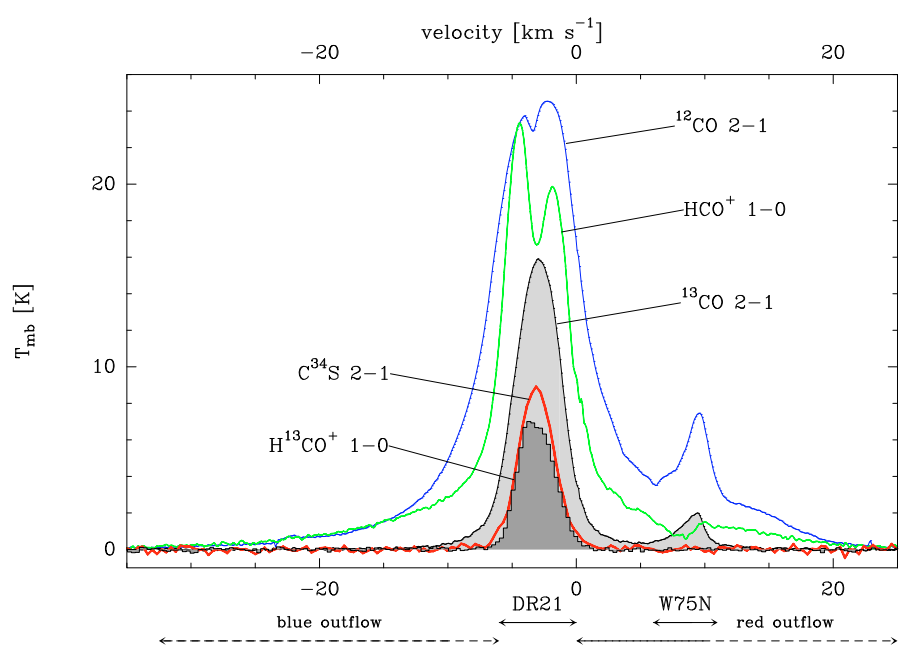

Fig. 4. Positionally averaged (across the whole observing region for each line) spectra of all lines observed in the DR21 filament using the IRAM $30 \mathrm{~m}$ telesope. The $\mathrm{HCO}^{+}$line is multiplied by a factor 8.5 , the $\mathrm{C}^{34} \mathrm{~S}$ and $\mathrm{H}^{13} \mathrm{CO}^{+}$lines by 10 .

\subsubsection{The DR21 filament in velocity-integrated maps}

Based on the average spectrum (Fig. 4) we produced line integrated maps of the bulk emission of the DR21 cloud (Fig. 5). Though there are differences in the detailed emission distribution, one can separate three main regions: a southern cloud with DR21, a middle emission peak with DR21(OH), and a northern extension with two secondary peaks.
While DR2 1 is most prominent in the $\mathrm{HCO}^{+}$and ${ }^{13} \mathrm{CO}$ lines, $\mathrm{DR} 21(\mathrm{OH})$ is more pronounced in all optically thin lines. A small shift in the position of peak emission of DR21(OH) can be discerned by comparing $\mathrm{C}^{34} \mathrm{~S} / \mathrm{H}^{13} \mathrm{CO}^{+}$and $\mathrm{N}_{2} \mathrm{H}^{+}$. For $\mathrm{N}_{2} \mathrm{H}^{+}$, we determined the optical depth using the hyperfine structure pattern of this line and found that $\tau$ remains below 1 basically everywhere in the map. The latter traces cold, dense gas that is located more south of the warmer central part of the DR21(OH) region. Mauersberger et al. (1986) report a temperature above $200 \mathrm{~K}$ in a small region of size $<9^{\prime \prime}$ in DR21(OH), and Wilson \& Mauersberger (1990) determine a rotation temperature of $34 \mathrm{~K}$ from $\mathrm{NH}_{3}$ observations. Mangum et al. (1991,1992) interpreted their observations as a detection of a B-star within the DR21 $(\mathrm{OH})$ clump. The northern part of the filament is more prominent in $\mathrm{N}_{2} \mathrm{H}^{+}$and $\mathrm{H}^{13} \mathrm{CO}^{+}$emission and not in $\mathrm{C}^{34} \mathrm{~S}$. Wilson \& Mauersberger (1990), and Wienen (2008) derive values around $20 \mathrm{~K}$ for this region.

Figure 6 is a position-velocity cut in ${ }^{12} \mathrm{CO}$ and ${ }^{13} \mathrm{CO} 2 \rightarrow 1$ and $\mathrm{HCO}^{+} 1 \rightarrow 0$ along the filament in which all data points in RA were averaged. The greyscale image of ${ }^{12} \mathrm{CO}$ emission shows that the bulk emission of the DR21 filament at $-3 \mathrm{~km} \mathrm{~s}^{-1}$ and the bulk emission of the W75N component at $9 \mathrm{~km} \mathrm{~s}^{-1}$ are apparently connected. The gas linking the two components has a low column density since it is only visible in ${ }^{12} \mathrm{CO}$ and not in ${ }^{13} \mathrm{CO}$ (red contours). A lack of ${ }^{12} \mathrm{CO}$ emission exists between $\Delta \delta=0^{\prime \prime}$ and $-150^{\prime \prime}$ at $\sim 8 \mathrm{~km} \mathrm{~s}^{-1}$, indicating that the (cooler) W75N cloud lies in front of the DR21 filament and causes the ${ }^{12} \mathrm{CO}$ self-absorption. Dickel et al. (1978) and Bieging et al. (1982) came to the same conclusion following their detection of self-absorbed line profiles in $\mathrm{H}_{2} \mathrm{CO}$ and $\mathrm{OH}$ toward DR21. However, the situation is slightly more complicated because the 


\section{N. Schneider et al.: DR21 filament}
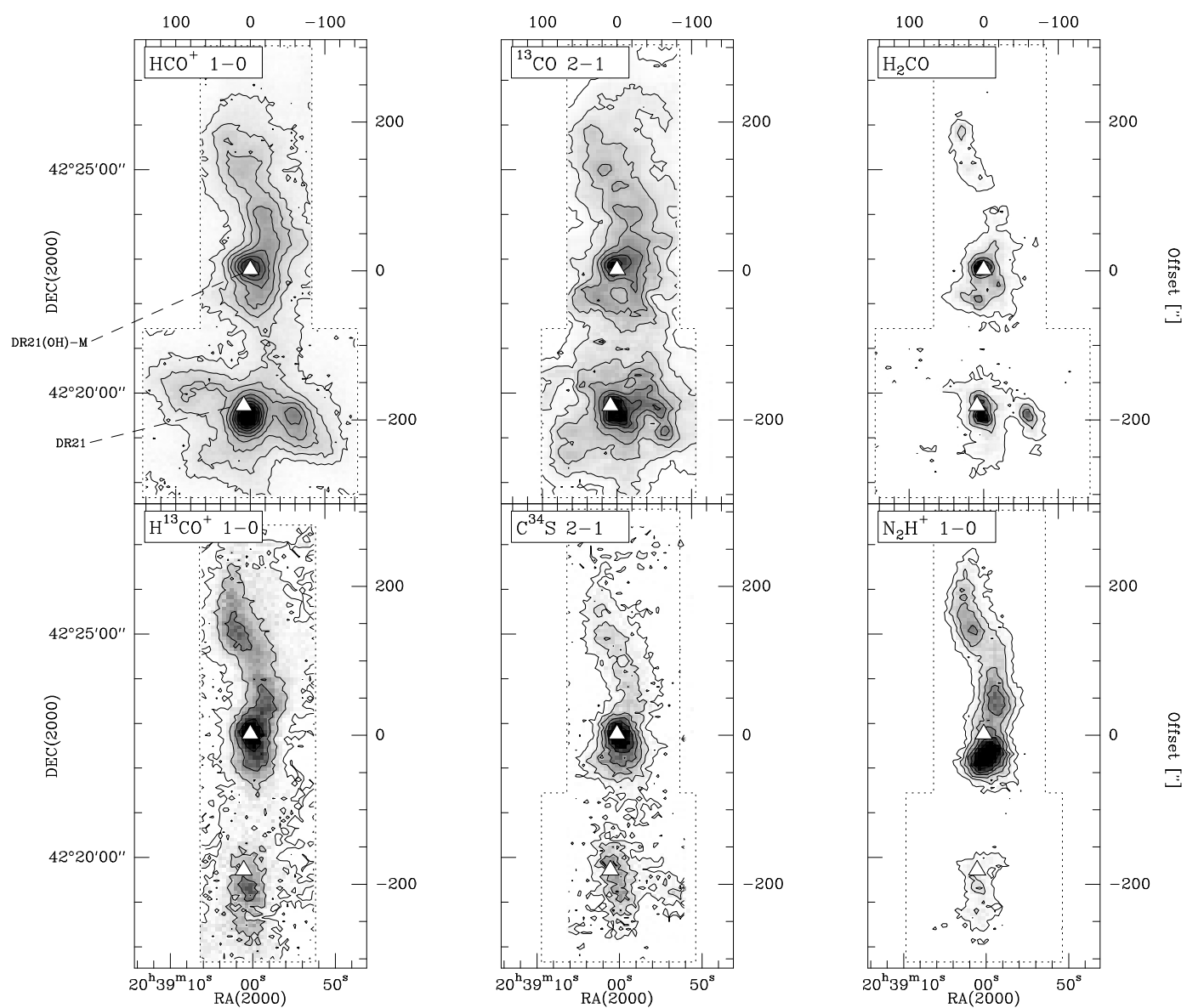

Fig. 5. Velocity-integrated spectral line maps of the DR21 filament. The $\mathrm{HCO}^{+} 1 \rightarrow 0$ and ${ }^{13} \mathrm{CO} 2 \rightarrow 1$ lines are integrated over a velocity range from -11 to $5 \mathrm{~km} \mathrm{~s}^{-1}$, the $\mathrm{H}_{2} \mathrm{CO} 3(1,2) \rightarrow 2(1,1)$ line from -11 to $2 \mathrm{~km} \mathrm{~s}^{-1}$, and all other lines from -7 to $1 \mathrm{~km} \mathrm{~s}^{-1}$. Contour levels follow the notation start/end/step with $15 \sigma=3.8 / 87.3 / 7.5 \mathrm{~K} \mathrm{~km} \mathrm{~s}^{-1}\left(\mathrm{HCO}^{+}\right), 6 \sigma=1.3 / 10.9 / 3.9 \mathrm{~K} \mathrm{~km} \mathrm{~s}^{-1}\left(\mathrm{H}^{13} \mathrm{CO}^{+}\right), 30 \sigma=34.1 / 249.4 / 23.9 \mathrm{~K} \mathrm{~km} \mathrm{~s}{ }^{-1}$ $\left({ }^{13} \mathrm{CO}\right), 6 \sigma=5.9 / 14.7 / 2.9 \mathrm{~K} \mathrm{~km} \mathrm{~s}^{-1}\left(\mathrm{C}^{34} \mathrm{~S}\right), 9 \sigma=6.4 / 49.1 / 10.7 \mathrm{~K} \mathrm{~km} \mathrm{~s}^{-1}\left(\mathrm{H}_{2} \mathrm{CO}\right), 3 \sigma=0.27 / 1.9 / 0.27 \mathrm{~K} \mathrm{~km} \mathrm{~s}^{-1}\left(\mathrm{~N}_{2} \mathrm{H}^{+}\right)$. Triangles indicate the positions of DR21(OH) and DR21.
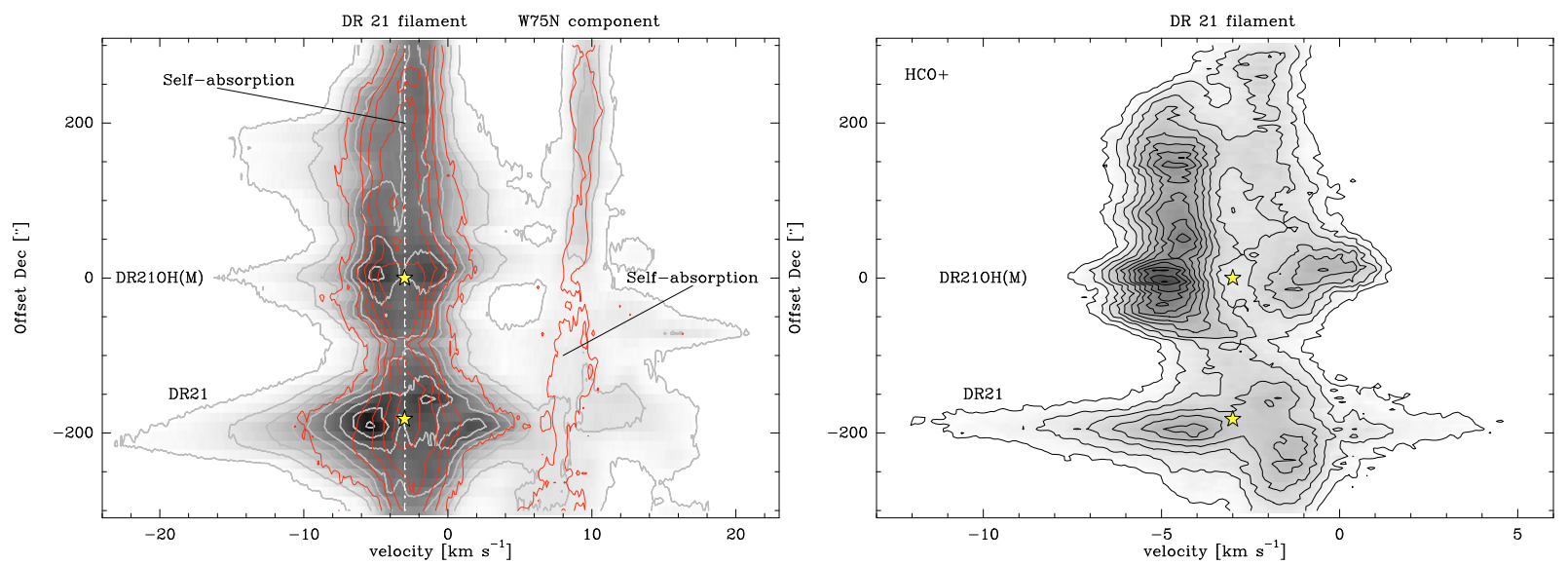

Fig. 6. Position-velocity cut along constant RA, i.e. along the DR21 filament. Left: ${ }^{12} \mathrm{CO} 2 \rightarrow 1$ (grey scale and grey contours) and ${ }^{13} \mathrm{CO} 2 \rightarrow 1$ (red contours). Contour levels are 2 to $34 \mathrm{~K} \mathrm{~km} \mathrm{~s}^{-1}$ by $2 \mathrm{~K} \mathrm{~km} \mathrm{~s}^{-1}$ for ${ }^{12} \mathrm{CO}$ and 1.5 to $16.5 \mathrm{~K} \mathrm{~km} \mathrm{~s}^{-1}$ by $3 \mathrm{~K} \mathrm{~km} \mathrm{~s}^{-1}$ for ${ }^{13} \mathrm{CO}$. The stars mark DR21(OH) and DR21. Right: the same plot for $\mathrm{HCO}^{+}$with contour levels from 1 to $8 \mathrm{~K} \mathrm{~km} \mathrm{~s}^{-1}$ by $0.5 \mathrm{~K} \mathrm{~km} \mathrm{~s}^{-1}$.

W75N line component is blended in its southern part $\left(<0^{\prime \prime}\right)$ with emission from the "Great Cygnus rift", which is basically not seen in ${ }^{13} \mathrm{CO}$ emission (Schneider et al. 2007). The rift is an extended, low-density gas cloud at a distance of around $600 \mathrm{pc}$. Its emission can start as low as $+4 \mathrm{~km} \mathrm{~s}^{-1}$ and makes it thus difficult to disentangle the different emission features. It is, however, possible that there is a real physical connection/interaction between the DR21 filament and the W75N cloud, as proposed by
Dickel et al. (1978). They suggested that the "W75N" cloud is differentially slowed down as it passes the edge of the DR21 cloud due to the observed N-S oriented velocity gradient from $+11 \mathrm{~km} \mathrm{~s}^{-1}$ to $+9 \mathrm{~km} \mathrm{~s}^{-1}$ (which is clearly seen in our $\mathrm{CO}$ data as well).

Several outflows are identified out of which the DR21 outflow at $\Delta \delta=-200^{\prime \prime}$ is the most pronounced. However, its red flow is not clearly defined because (i) it is blended with the 

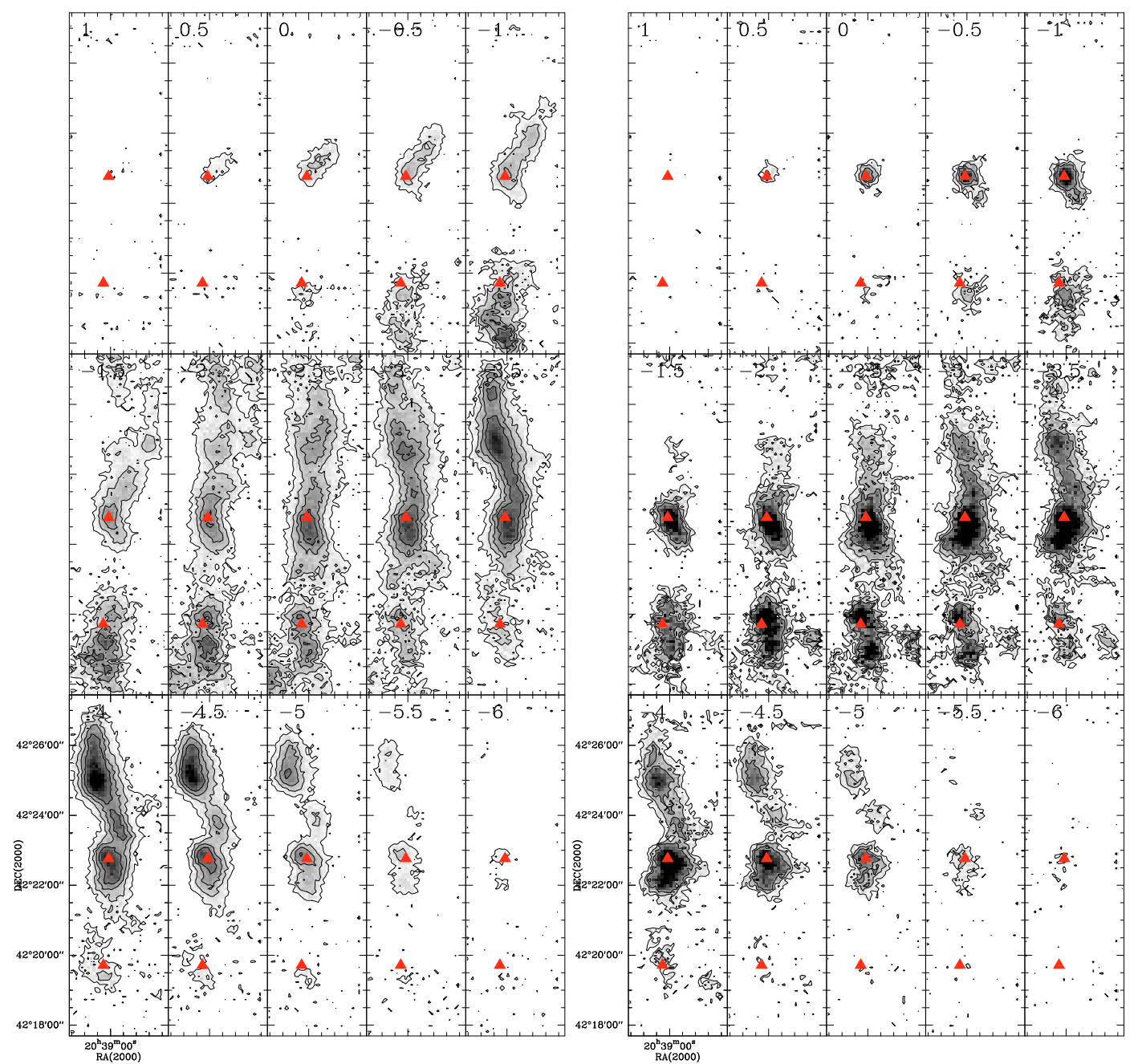

Fig. 7. Channel maps of $\mathrm{H}^{13} \mathrm{CO}^{+} 1 \rightarrow 0$ (left) and $\mathrm{C}^{34} \mathrm{~S} 2 \rightarrow 1$ emission (right) between $+1 \mathrm{~km} \mathrm{~s}^{-1}$ and $-6 \mathrm{~km} \mathrm{~s}^{-1}$. The red triangles indicate the positions of DR21(OH) (north) and DR21 (south), respectively.

"Great Rift" emission and (ii) because it runs into an HII region (Garden et al. 1992). The blue flow, however, is clearly defined. Other "flow-features" are identified at $\Delta \delta=0$ " (DR21OH(M)) and at $\sim 180^{\prime \prime}$, both look well-defined at negative velocities and disturbed for positive velocities. The position-velocity diagram for $\mathrm{HCO}^{+}$shows that these outflows are much less obvious than that towards DR21. We come back to outflows in Sect. 4.3 and in particular the one seen at DR21OH(M) in Sects. 4.2.3 and 5.2.

Another feature visible in Fig. 6 is a region of self-absorption in ${ }^{12} \mathrm{CO}$ (emission regions in ${ }^{13} \mathrm{CO}$ ) at around $-3 \mathrm{~km} \mathrm{~s}^{-1}$ along the whole filament (indicated by a dashed white line). This central region of self-absorption is even more clearly identified in the $\mathrm{HCO}^{+}$map. The strongest self-absorption is found at the declination offsets of DR21 and DR21OH(M). We discuss this finding in more detail in Sect. 5.2.

\subsubsection{A subfilament at $0 \mathrm{~km} \mathrm{~s}^{1}-$ channel maps of optically thin lines}

Figure 7 shows channel maps of $\mathrm{H}^{13} \mathrm{CO}^{+}$and $\mathrm{C}^{34} \mathrm{~S}$ emission between +1 and $-6 \mathrm{~km} \mathrm{~s}^{-1}$. The distribution of bulk emission of the cloud between -2 and $-5 \mathrm{~km} \mathrm{~s}^{-1}$ is similar in both tracers and follows the typical NS-ridge of the DR21 filament. Some emission peaks are stronger in $\mathrm{H}^{13} \mathrm{CO}^{+}$than in $\mathrm{C}^{34} \mathrm{~S}$ (and vice versa) due to the different density and temperature regimes. An example is seen at velocity $-4 \mathrm{~km} \mathrm{~s}^{-1}$ where $\mathrm{C}^{34} \mathrm{~S}$ peaks prominently at the DR21(OH) clump while $\mathrm{H}^{13} \mathrm{CO}^{+}$emits more strongly in the northern part of the filament.

At velocities larger than $-2 \mathrm{~km} \mathrm{~s}^{-1}$, we identify an extended emission component between -1 and $0.5 \mathrm{~km} \mathrm{~s}^{-1}$ that appears prominent in $\mathrm{H}^{13} \mathrm{CO}^{+}$(and $\mathrm{N}_{2} \mathrm{H}^{+}$not shown here) but not in $\mathrm{C}^{34} \mathrm{~S}$ (and $\mathrm{H}_{2} \mathrm{CO}$ also not shown here) northwest of DR21(OH). In ${ }^{12} \mathrm{CO}$ and ${ }^{13} \mathrm{CO} 2 \rightarrow 1$, this feature is discernible as well, but largely blended with other emission components. This subfilament is the eastern end of the large-scale filament F3 that was observed in lower-angular resolution ${ }^{13} \mathrm{CO} 1 \rightarrow 0$ channel maps (Fig. 3). It was also seen in isotopomeric $\mathrm{CO} 3 \rightarrow 2$ data (Vallée \& Fiege 2006) and interpreted by the authors as being caused by outflow emission from DR21OH(M). An outflow is indeed detected at this position (see Sect. 4.3) but the emission in the velocity range $\sim-1$ to $1 \mathrm{~km} \mathrm{~s}^{-1}$ is clearly due to an individual cloud fragment. Spectra towards a position within this fragment are shown in Fig. 8. An individual Gaussian line is seen at $0 \mathrm{~km} \mathrm{~s}^{-1}$ for all optically thin lines (at this position rather close to $\mathrm{DR} 21 \mathrm{OH}(\mathrm{M})$ also for $\left.\mathrm{C}^{34} \mathrm{~S}\right)$. It becomes obvious that the ${ }^{12} \mathrm{CO} 2 \rightarrow 1$ line - even at an angular resolution of $11^{\prime \prime}-$ is not useful for separating individual components since it is too sensitive to low-density emission. Figure 9 superimposes $\mathrm{N}_{2} \mathrm{H}^{+}$ and $\mathrm{H}^{13} \mathrm{CO}^{+}$emission in the two major velocity ranges, the bulk emission between -6 to $-3 \mathrm{~km} \mathrm{~s}^{-1}$ in grey scale and the fragment 


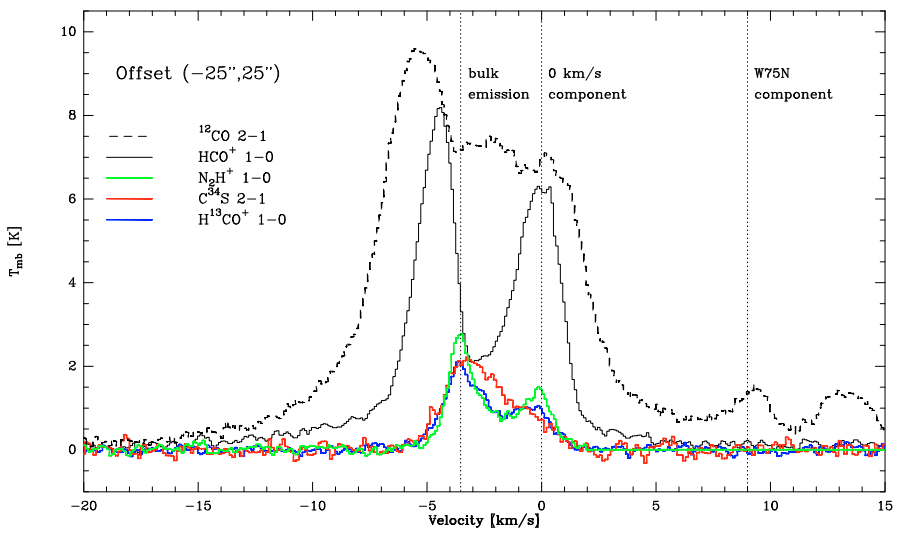

Fig. 8. Spectra of several line tracers at the position $-25^{\prime \prime}, 25^{\prime \prime}$. The ${ }^{12} \mathrm{CO}$ line was reduced by a factor 3.5 and for the $\mathrm{N}_{2} \mathrm{H}^{+}$line, we only show the 101-012 component of the hyperfine structure (to avoid confusion). The different emission components are indicated with short dashed lines.

emission between -1 and $1 \mathrm{~km} \mathrm{~s}^{-1}$ as overlaying contours. The emission in $\mathrm{H}^{13} \mathrm{CO}^{+}$for both velocity ranges peaks at the position of DR21OH-(M). A secondary weaker peak is found $\sim 50^{\prime \prime}$ further south, close to N48 (or DR21OH-(S)) ${ }^{6}$, where $\mathrm{N}_{2} \mathrm{H}^{+}$ between -6 and $-3 \mathrm{~km} \mathrm{~s}^{-1}$ has its peak emission. This supports the "hot core" scenario for DR21-OH(M) because the gas there is warmer and chemically richer than in the dense, cold core of DR21OH-(S). This core, however, is embedded in a warmer envelope traced by $\mathrm{H}^{13} \mathrm{CO}^{+}$. Even more interesting is the $0 \mathrm{~km} \mathrm{~s}^{-1}$ component. The emission distribution in $\mathrm{H}^{13} \mathrm{CO}^{+}$ and $\mathrm{N}_{2} \mathrm{H}^{+}$suggests that this fragment "falls" on the densest part of the DR21OH(M) clump. The $\mathrm{N}_{2} \mathrm{H}^{+}$peak is more distinct and smaller in extent and shifted with respect to $\mathrm{H}^{13} \mathrm{CO}^{+}$, again indicating that $\mathrm{H}^{13} \mathrm{CO}^{+}$emission arises from the warm envelope.

\subsection{Ouflows}

Figure 10 shows in more detail the outflow features detected in Fig. 6. From the position-velocity plot and positionally averaged ${ }^{12} \mathrm{CO}$ spectra, we can clearly define the blue emission range between -30 and $-17 \mathrm{~km} \mathrm{~s}^{-1}$. The redshifted emission is more difficult to discern because at higher positive velocities, a part of the line emission in the southern part of the DR21 filament is due to the "Great Cygnus rift" (see Sect. 4.2.2). However, the velocity range $\sim 17$ to $\sim 30 \mathrm{~km} \mathrm{~s}^{-1}$ characterizes well the red wing.

Several blue outflow sources are clearly recognized. These are all associated with mm-continuum sources, i.e. N53, N44, and N45. Source N51 shows broad line wings in ${ }^{12} \mathrm{CO}$ and $\mathrm{HCO}^{+}$(Fig. 14), which is most likely only the south extention of the N53 outflow. Southeast of N44 and at offsets $-100^{\prime \prime},-140^{\prime \prime}$ are clearly defined blue sources that have no red counterparts. Sources N53, N48, and N44 show outflow emission in SiO $2 \rightarrow$ 1 (Motte et al. 2007) with a prominent red wing for N53 and a blue wing for N44. They are classified as massive infrared-quiet protostellar cores (Motte et al. 2007) with masses of 85, 197, and $446 M_{\odot}$, respectively. N45 exhibits no $\mathrm{SiO}$ emission but is associated with an $\mathrm{H}_{2}$ jet (source A 3-1 in Davis et al. 2007), which is probably driven by a low- or intermediate-mass YSO (all $\mathrm{H}_{2}$-jets are marked with crosses in the plot). N44 is the known outflow

\footnotetext{
${ }^{6}$ In this paper, we follow the notation of mm-continuum clumps given in Motte et al. (2007). This source list is more complete than the one from Chandler et al. (1993). However, for clarity, we still give the former names DR21OH(Main, South, West) on occasion.
}
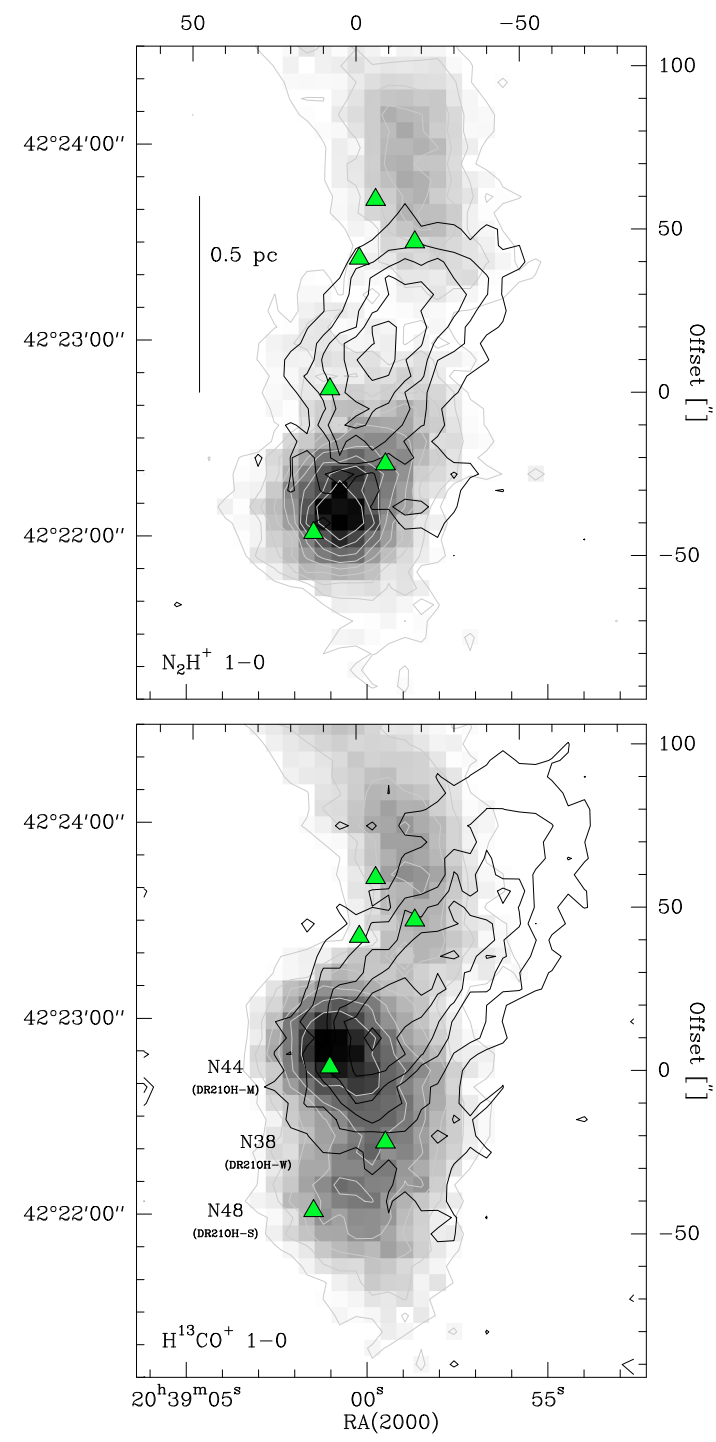

Fig. 9. Maps of line integrated $\mathrm{N}_{2} \mathrm{H}^{+} 1 \rightarrow 0($ top $)$ and $\mathrm{H}^{13} \mathrm{CO}^{+} 1 \rightarrow 0$ (bottom) emission of the DR21(OH) region. For $\mathrm{N}_{2} \mathrm{H}^{+}$, we integrate over all hyperfine structure components, the greyscale covering the velocity range -6 to $-3 \mathrm{~km} \mathrm{~s}^{-1}$ going from 0.8 to $6.7 \mathrm{~K} \mathrm{~km} \mathrm{~s}^{-1}$ and the black contours covering the range -1 to $1 \mathrm{~km} \mathrm{~s}^{-1}$ and go from 0.32 to 1.93 in steps of $0.32 \mathrm{~K} \mathrm{~km} \mathrm{~s}^{-1}$. For $\mathrm{H}^{13} \mathrm{CO}^{+}$the same velocity range -6 to $-3 \mathrm{~km} \mathrm{~s}^{-1}$ represents grey scale $\left(0.64\right.$ to $\left.3.8 \mathrm{~K} \mathrm{~km} \mathrm{~s}^{-1}\right)$ and the black contours the velocity range -1 to $1 \mathrm{~km} \mathrm{~s}^{-1}$ with contours from 0.67 to 6.6 by $0.54 \mathrm{~K} \mathrm{~km} \mathrm{~s}^{-1}$. Green triangles mark mm-continuum sources, following the notation of Motte et al. (2007).

of DR21(OH), seen in CS $5 \rightarrow 4$ emission (Richardson et al. 1994) and indirectly traced by the spatial and kinematic distribution of maser sources (Plambeck \& Menten 1990). However, no $\mathrm{H}_{2}$ emission was detected (Davis et al. 2007).

This source is well studied (e.g. Garden et al. 1991a,b, 1992), so we do not go into details here. In our map, we see also the classical east-west orientation of the flow with some north-south features (e.g. component " $\mathrm{C}$ " in Garden et al.). Among the other sources, only N53 and N44 show red wing emission. It is not clear what the emission feature running east-west at offsets $60^{\prime \prime}$ in declination is. In Fig. 10, it shows up in $\mathrm{HCO}^{+}$emission as a typical outflow feature. But it has no blue counterpart and may well be associated with the Cygnus Rift. 


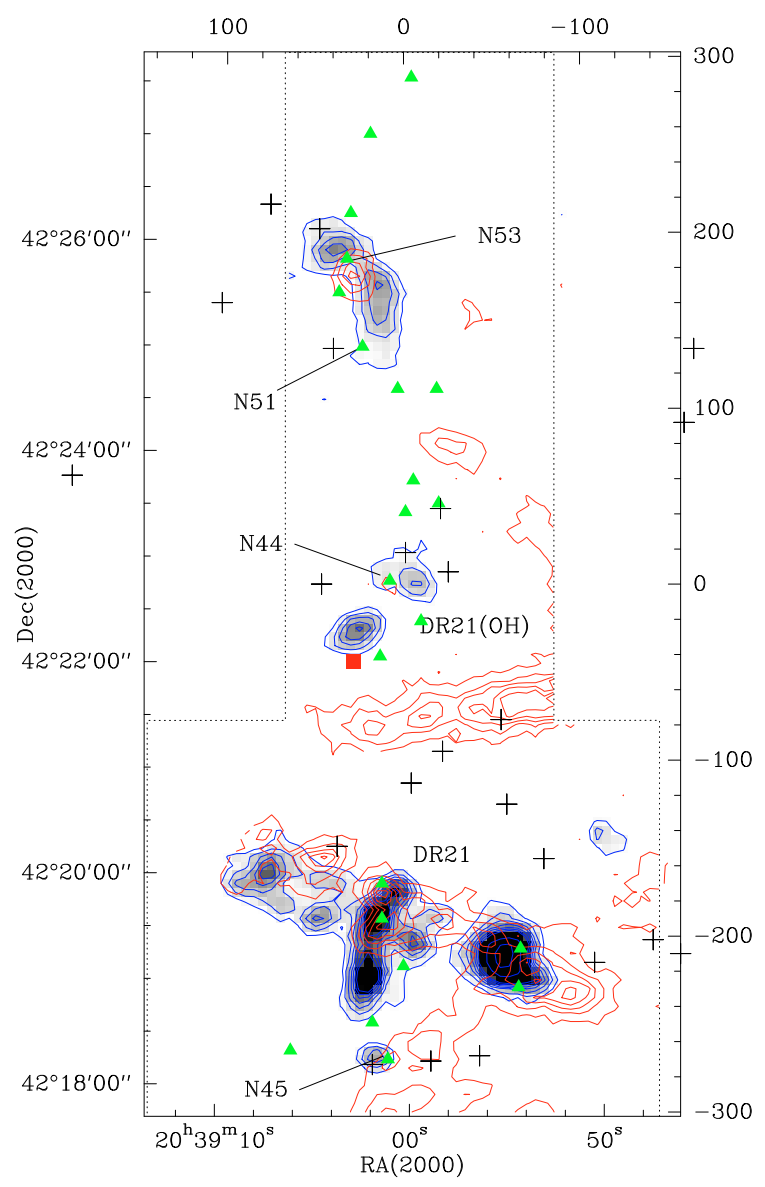

Fig. 10. Map of line integrated ${ }^{12} \mathrm{CO} 2 \rightarrow 1$ emission in the DR21 filament. Blue contours indicate a velocity range of -30 to $-17 \mathrm{~km} \mathrm{~s}^{-1}$ (levels 12 to 60 by $8 \mathrm{~K} \mathrm{~km} \mathrm{~s}^{-1}$ and then $100,140 \mathrm{~K} \mathrm{~km} \mathrm{~s}^{-1}$ ), red contours a range of 17 to $30 \mathrm{~km} \mathrm{~s}^{-1}$ (levels 6 to 36 by $6 \mathrm{~K} \mathrm{~km} \mathrm{~s}^{-1}$ ). Green triangles indicate mm-continuum sources from Motte et al. (2007), black crosses mark $\mathrm{H}_{2}$ jets and knots discovered by Davis et al. (2007), and the red square south of DR21-OH indicates the "Extremely Red Object" ERO1 detected by Marston et al. (2004).

\section{Analysis}

This section is devoted to an analysis of the physical properties of the DR21 filament. We, however, exclude the area around DR21 since this region is perturbed by outflow emission and line confusion. We focus instead on the northern part of the DR21 filament, including the region of DR21(OH).

\subsection{Physical properties of the sub-filaments}

Our molecular line observations show that the DR21 filament is connected to several sub-filaments seen on large and small scales. In the ${ }^{13} \mathrm{CO} 1 \rightarrow 0$ channel maps (Fig. 3), it becomes obvious that the most prominent filament F3 covers a velocity range of $\sim 3 \mathrm{~km} \mathrm{~s}^{-1}$ (between $-4 \mathrm{~km} \mathrm{~s}^{-1}$ and $-1 \mathrm{~km} \mathrm{~s}^{-1}$ ) and has a projected length of $\sim 7 \mathrm{pc}$ (the width is $\sim 1.5 \mathrm{pc}$ ). The channel maps show that the top of the column lies at higher velocities, indicating that this part of the filament is tilted away from the observer. Assuming an average angle to the line-of-sight of $57.3^{\circ}$ (in the case of a random distribution of orientation angles, the average angle is equal to this value), this implies a dynamic lifetime of $\sim 1.5 \times 10^{6} \mathrm{yr}$. The mass of the filament (for more details see Appendix A about the mass determination from ${ }^{13} \mathrm{CO}$ data) is $2600 M_{\odot}$ and the average density is $690 \mathrm{~cm}^{-3}$. This sub-filament indeed corresponds to "clump 7" in the DR21 region, identified in the ${ }^{13} \mathrm{CO} 2 \rightarrow 1$ survey by Schneider et al. (2006).

The higher angular resolution IRAM observations resolve the detailed structure and show how this sub-filament connects to the DR21(OH) clump within the filament (see Sect. 4.2.3). The mass, determined from the line integrated $\mathrm{H}^{13} \mathrm{CO}^{+}$intensity (see Appendix A), is $1320 M_{\odot}$ with an average density of $8.1 \times 10^{4} \mathrm{~cm}^{-3}$. This mass estimate, however, is rather uncertain because of the uncertain $\left[\mathrm{HCO}^{+}\right] /\left[\mathrm{H}_{2}\right]$ abundance ratio. From the channel maps (Fig. 7), we see that the filament extends up to $\sim 0.5 \mathrm{~km} \mathrm{~s}^{-1}$ and therefore has a total radial velocity range of $4.5 \mathrm{~km} \mathrm{~s}^{-1}$ at a length of $\sim 1 \mathrm{pc}$. If we assume that it connects to the large filament F3, the lower velocity levels are equally around $-4 \mathrm{~km} \mathrm{~s}^{-1}$, thus implying a total velocity range of $4.5 \mathrm{~km} \mathrm{~s}^{-1}$. Again, assuming an average angle to the lineof-sight of $57.3^{\circ}$, this implies a very short dynamic lifetime of $\sim 1.4 \times 10^{5} \mathrm{yr}$.

In addition, we note that in the high spatial resolution IRAM data, this sub-filament shows a position angle of $\sim 45^{\circ}$ in projection to the sky, while on a larger scale F3 is mostly eastwest oriented $\left(\mathrm{PA} \sim 100^{\circ}\right)$. This bend, which could be related to the deep gravitational well of the DR21(OH) clump, may also be present along the line-of-sight, explaining the apparent acceleration from 3 to $4.5 \mathrm{~km} \mathrm{~s}^{-1}$ along the sub-filament between large and small scales. Assuming a constant velocity of $4.5 \mathrm{~km} \mathrm{~s}^{-1}$ (the largest observed range of velocity) along the whole F3 sub-filament, the angle of the sub-filament to the lineof-sight would be $48^{\circ}$ before the bend and $90^{\circ}$ after the bend, close to DR21 $(\mathrm{OH})$ (i.e. streaming away from the observer along the line-of-sight). This 3D view of F3 seems to be corroborated by the largest velocities in the IRAM data being found closest to DR21 $(\mathrm{OH})$. The total length of the F3 sub-filament would then be at least $9.4 \mathrm{pc}\left(7 \mathrm{pc}\right.$ divided by $\left.\sin \left(48^{\circ}\right)\right)$ with no information about the length along the line-of-sight after the bend, leading to a total dynamic timescale of $\sim 2.1 \times 10^{6} \mathrm{yr}$, and an input mass rate of $\sim 1.9 \times 10^{-3} M_{\odot} \mathrm{yr}^{-1}$ (total mass of F3 on large and small scales divided by the timescale).

\subsection{Infall signatures across the filament}

Figure 11 shows an overlay of $\mathrm{HCO}^{+} 1 \rightarrow 0$ spectra (red) on a greyscale plot of line integrated $\mathrm{N}_{2} \mathrm{H}^{+} 1 \rightarrow 0$ emission (both at an angular resolution of $\sim 30^{\prime \prime}$ ). It is obvious that $\mathrm{HCO}^{+}$displays along the whole filament the "typical" profile of an optically thick spectral line which is indicative of infalling gas, i.e. a blue-shifted line wing that is stronger than the red wing (Myers et al. 1996). This self-absorption was already visible in ${ }^{12} \mathrm{CO}$ emission (Fig. 6) but becomes more pronounced using the $\mathrm{HCO}^{+}$line. In order to quantify this infall signature, we determined the normalized integrated intensity (see Fig. 12) between the blue and red line components (with $T$ (blue) $>T$ (red)), i.e. the "amount" of self-absorption, and plotted this quantity in Fig. 13. It is remarkable that self-absorption is seen everywhere in the filament on a size scale of $\sim 10^{\prime}=5 \mathrm{pc}$ with smooth transitions and not only locally at the position of mm-continuum sources. However, spectra with strongest self-absorption are found at the position of N44 (DR21OH(M)).

To exclude the possibility that we observe several line components, we display in Fig. 14 examples of various lines at three selected positions (that are modeled in Sect. 5.3). First, the spectra show that ${ }^{12} \mathrm{CO}$ is not the most reliable infall tracer since it is ubiquitous and the infall profile is most clearly seen in $\mathrm{HCO}^{+}$. The figure also clearly proves that not two different cloud 


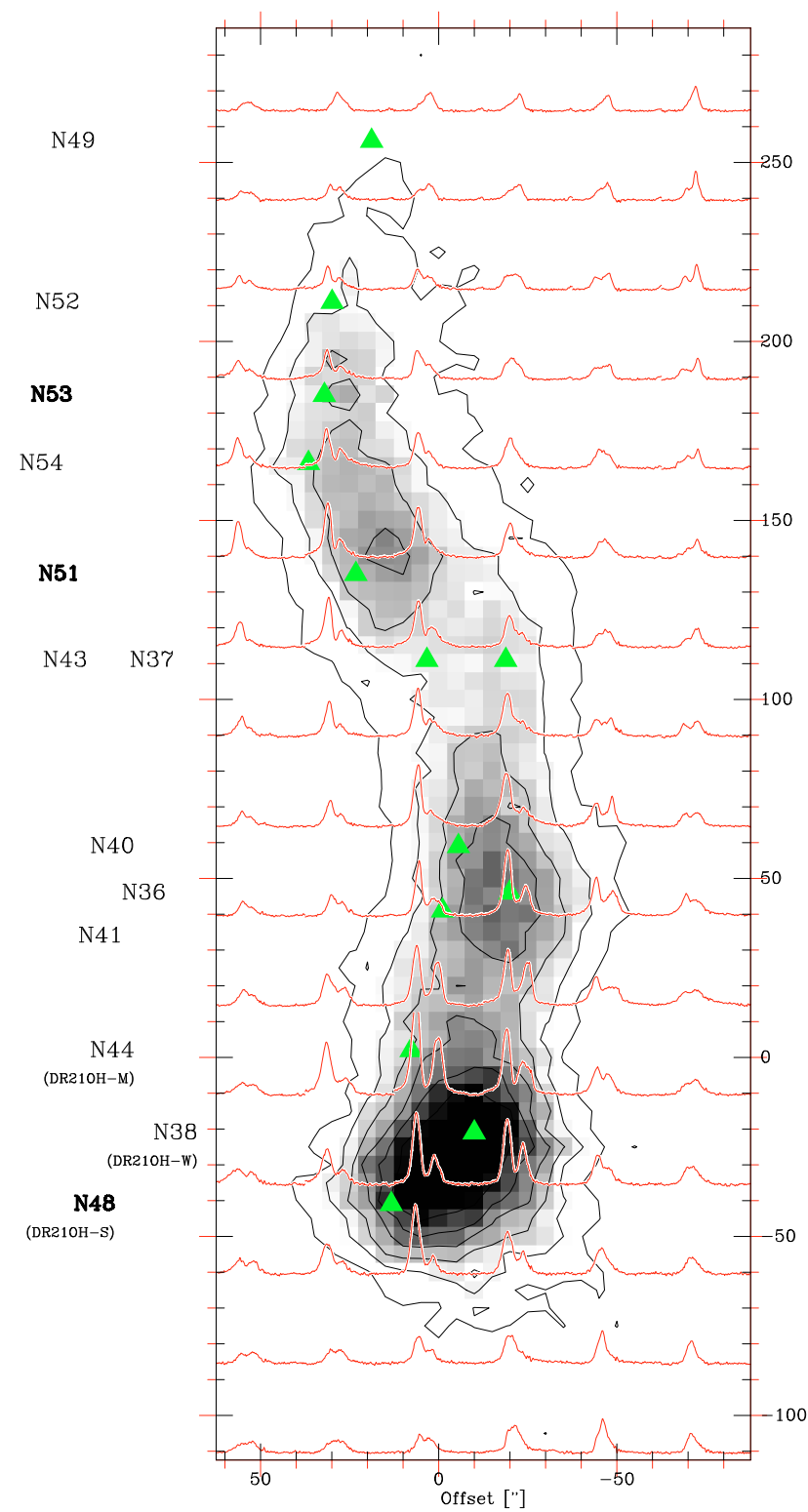

Fig. 11. Greyscale map of line integrated $\mathrm{N}_{2} \mathrm{H}^{+} 1 \rightarrow 0$ emission of the DR21 filament with spectra of $\mathrm{HCO}^{+}$overlaid in red. The spectra cover a velocity range of -10 to $10 \mathrm{~km} \mathrm{~s}^{-1}$ and a temperature range of -1 to $12 \mathrm{~K}$. Green triangles indicate $\mathrm{mm}$-continuum sources from Motte et al. (2007) and are named accordingly. N44, N38, and N48 coincide with the mm-sources DR21OH-M (Main), W (West), and S (South), respectively, named by Chandler et al. (1993). The $0^{\prime \prime}, 0^{\prime \prime}$ position is DR21OH(M) (see Sect. 3). Spectra of the sources in bold (N53, N51, and N48) are displayed in Fig. 14.

components are observed since all optically thin lines $\left(\mathrm{C}^{34} \mathrm{~S}\right.$, $\mathrm{N}_{2} \mathrm{H}^{+}, \mathrm{H}^{13} \mathrm{CO}^{+}$) peak in the emission gap of $\mathrm{HCO}^{+}$. Apart from the known line components at $-3 \mathrm{~km} \mathrm{~s}^{-1}$ (bulk emision of the DR21 filament) and $+9 \mathrm{~km} \mathrm{~s}^{-1}$ (W75N, only visible in ${ }^{12} \mathrm{CO}$ $2 \rightarrow 1$ ), the $0 \mathrm{~km} \mathrm{~s}^{-1}$ component (see Sect. 4.2.3) is best traced in $\mathrm{N}_{2} \mathrm{H}^{+}$emission.

Since it is assured that we observe a true infall signature across the DR21 filament, we determined the infall velocity $v_{\text {in }}$ to first order using the method described in Myers et al. (1996).

$v_{\text {in }}=\frac{\sigma^{2}}{v_{\text {red }}-v_{\text {blue }}} \ln \frac{1+e\left(T_{\mathrm{BD}} / T_{\mathrm{D}}\right)}{1+e\left(T_{\mathrm{RD}} / T_{\mathrm{D}}\right)}$

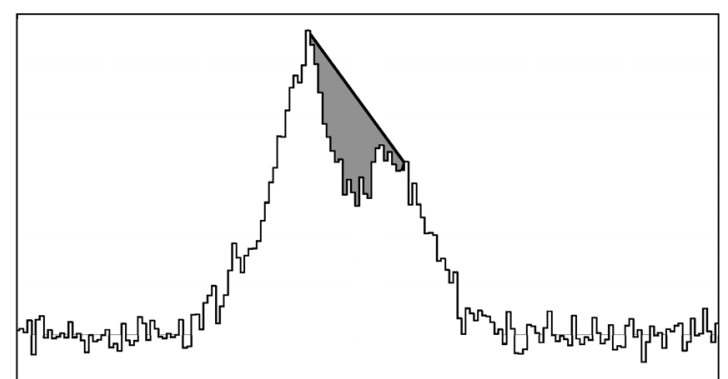

Fig. 12. The grey area, divided by the total line-integrated intensity over all velocities (the "normalized line intensity"), is shown in Fig. 13. Note that only spectra with a real infall line profile $(T$ (blue $)>T($ red $)$ ) were considered.

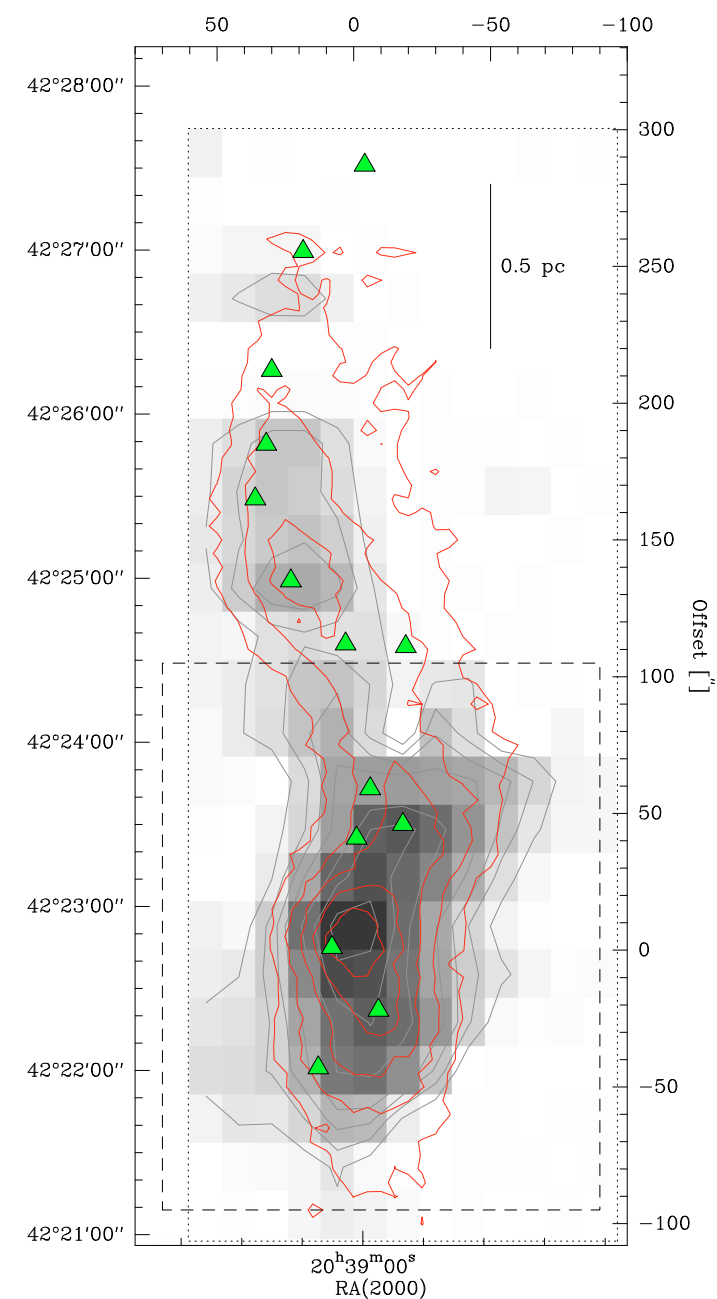

Fig. 13. Greyscale map of the ratio of self-absorption to line-integrated $\mathrm{HCO}^{+} 1 \rightarrow 0$ emission in the DR21 filament. Overlaid on that are red contours of $\mathrm{H}^{13} \mathrm{CO}^{+} 1 \rightarrow 0$ emission (contours go from 3 to $13 \mathrm{~K} \mathrm{~km} \mathrm{~s}^{-1}$ by $1.5 \mathrm{~K} \mathrm{~km} \mathrm{~s}^{-1}$ ). Green triangles indicate mm-continuum sources. The rectangle indicates the zoom region displayed in Fig. 9.

here, $\sigma$ is the $\mathrm{H}^{13} \mathrm{CO}^{+}$velocity dispersion, $T_{\mathrm{RD}}, v_{\text {red }}$ and $T_{\mathrm{BD}}$, $v_{\text {blue }}$ the temperature and the velocity of the red and blue part of the absorption profile, respectively, $e=2.71828$ the Eulerian number, and $T_{\mathrm{D}}$ is the brightness temperature of the dip. In all calculations, we excluded the area containing DR21 $\left(\Delta \delta<-100^{\prime \prime}\right)$. The velocity dispersion is determined from the FWHM line width of the optically thin $\mathrm{H}^{13} \mathrm{CO}^{+}$line $(\sigma=$ $\left.F W H M\left(\mathrm{H}^{13} \mathrm{CO}^{+}\right) / \sqrt{8 \ln (2)}\right)$. We disentangle the optically thin 

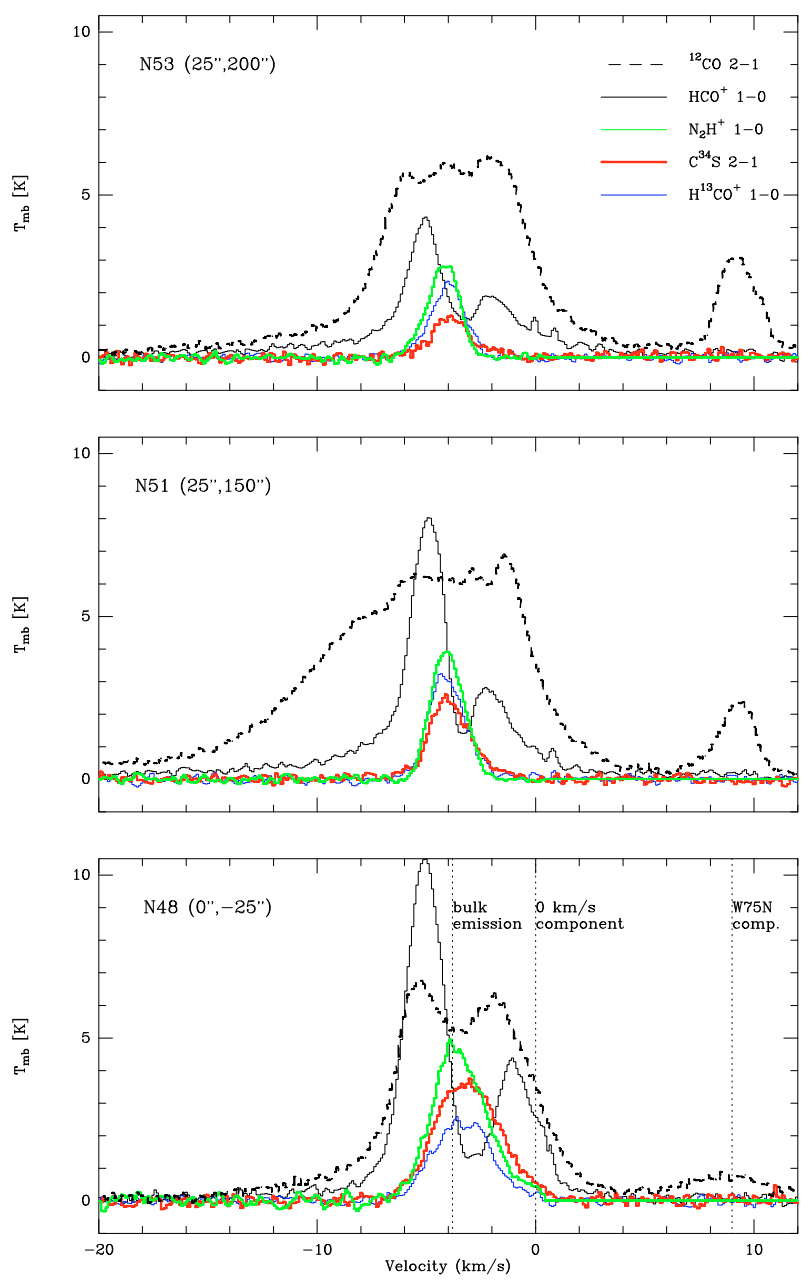

Fig. 14. Spectra of optically thick $\left({ }^{12} \mathrm{CO} 2 \rightarrow 1\right.$ and $\left.\mathrm{HCO}^{+} 1 \rightarrow 0\right)$ and thin $\left(\mathrm{N}_{2} \mathrm{H}^{+} 1 \rightarrow 0, \mathrm{C}^{34} \mathrm{~S} 2 \rightarrow 1\right.$, and $\left.\mathrm{H}^{13} \mathrm{CO}^{+}\right)$spectra from selected positions in the DR21 filament. The ${ }^{12} \mathrm{CO}$ intensity was reduced by a factor of 4 . To avoid confusion, the lower frequency HFS components of the $\mathrm{N}_{2} \mathrm{H}^{+}$line were suppressed.

emission of the molecular ridge $\left(\sim-3 \mathrm{~km} \mathrm{~s}^{-1}\right)$ from the infalling sub-filament at $0 \mathrm{~km} \mathrm{~s}^{-1}$ with a double Gaussian fit that precisely determines $\sigma$ of the bulk emission to be $-3 \mathrm{~km} \mathrm{~s}^{-1}$. For the optically thick $\mathrm{HCO}^{+}$line, we did not perform such a double Gaussian fit since the $-3 \mathrm{~km} \mathrm{~s}^{-1}$ component, showing the infall signature, dominates strongly over the single Gaussian $0 \mathrm{~km} \mathrm{~s}^{-1}$ component (this means that the subfilament does not show an infall signature). We determined the temperature and the velocity of the red $\left(T_{\mathrm{RD}}, v_{\text {red }}\right)$ and blue $\left(T_{\mathrm{BD}}, v_{\mathrm{blue}}\right)$ part of the absorption profile by fitting a Gaussian to each wing. We only computed the infall speed in a region where the emission of the second component does not contribute significantly and confuse the absorption profile. We note that we consider each point in the map as a single two-layer model (as outlined in Myers et al. 1996).

Figure 15 shows the results of this procedure. Supersonic infall velocities, $v_{\text {in }}>0.2 \mathrm{~km} \mathrm{~s}^{-1}$ for gas of a temperature of $10 \mathrm{~K}$ (typical value found), are detected within the red contour line, i.e. covering a large part of the filament. Interestingly, the highest values of $v_{\text {in }}\left(\sim 0.6-0.8 \mathrm{~km} \mathrm{~s}^{-1}\right)$ are not detected exactly towards the column density maxima (indicated by $\mathrm{N}_{2} \mathrm{H}^{+}$and $\mathrm{mm}-$ continuum emission in the right panels of Fig. 15), but, instead, show some significant spatial offsets ranging from $20^{\prime \prime}$ to $30^{\prime \prime}$ (i.e. of the order of $0.2 \mathrm{pc}$ ) in projection. In the center region (DR21OH(M) and N40) and in the south of the filament (N38 and N48), high values of $v_{\text {in }}$ are mainly due to the increasing optical depth $\tau$ of the $\mathrm{HCO}^{+}$line since the infall velocity is determined by the ratio $T_{\mathrm{BD}} / T_{\mathrm{RD}}$ (Eq. (1)), which increases with $\tau$. In the north, higher values of $v_{\text {in }}$ are offset by $\sim 20^{\prime \prime}$ to the dense clump containing N51 to N54 and are mainly related to the larger linewidths of the $\mathrm{N}_{2} \mathrm{H}^{+}$line (see Fig. 18, showing the same increase in linewidth for $\mathrm{H}^{13} \mathrm{CO}^{+}$displaced with respect to the dense clump).

Please note that this behaviour does not involve a gradual increase in infall speed since we only determine the infall velocity at each point of the map assuming local infall at each point. From this figure, we can not extract the radial profile of the infall speed within the whole filament. For that, we need a more realistic radiative transfer model, by considering the density and temperature structure in the dense clumps of the filament as we present in the next section.

\subsection{Radiative transport modelling with Simline}

We used the 1D non-LTE radiative transfer code Simline (Ossenkopf et al. 2001) to simultaneously model the observed optically thin $\mathrm{H}^{13} \mathrm{CO}^{+}$and optically thick $\mathrm{HCO}^{+}$lines of the two clumps indicated on the right panel of Fig. 15. These were extracted from the mm-continuum survey obtained by Motte et al. (2007) and characterize well the two major regions of infall. The southern Clump-14 is associated with DR21(OH) and contains the cores N36, N38, N41, N44, and N48. In the northern part of the filament, Clump-16 contains the cores N37, N43, N51, N53, and N54. The advantage of using mm-continuum data is that we have a rather reliable mass estimate that can be used as input to the model. Our ultimate goal is to derive the infall velocity and the level of depletion by constraining a simple physical model for these clumps.

\subsubsection{Input parameters and assumptions}

For both clumps, we used the Gaussian parameters (flux and FWHM) determined by Motte et al. (2007) and assumed the more realistic $\rho(r) \propto r^{-2}$ density profile to estimate the $90 \%$ mass and corresponding radius. We also recalculated the mass down to the level of $10 \%$ of the peak contour Clump-16 taking a mass-averaged temperature of $20 \mathrm{~K}$ (rather than the median temperature of Cygnus X clumps, $15 \mathrm{~K}$, used by Motte et al. 2007), which is in closer agreement with $\mathrm{NH}_{3}$ measurements of this clump (Wienen 2008). The model self-consistently takes the mass corresponding to the mass-averaged temperature with a profile $T(r)=T_{\text {in }}\left(r / r_{\text {in }}\right)^{\beta}$, where $\beta$ is between -0.2 and -0.6 ( $T_{\text {in }}$ and $r_{\text {in }}$ are the temperature and radius of the inner layer, see below). We adapted a two-layer geometry for the model since runs with only one layer did not reproduce well the observed line profiles, a result similar to that of Jakob et al. (2007) who equally applied a two-layer model for their low-angular resolution mid-J CO and atomic CI lines. However, they modeled their observations without considering molecular depletion and no or only very low infall speeds. We applied a subthermal external layer at constant temperature with an average density of $n \sim 10^{3}-10^{4} \mathrm{~cm}^{-3}$ because the self-absorption dip is very deep.

For the determination of the turbulent line-width, we used the optically thin line of $\mathrm{H}^{13} \mathrm{CO}^{+}$. A single Gaussian fit yielded line-widths of $\sim 2.5 \pm 0.5 \mathrm{~km} \mathrm{~s}^{-1}$ for Clump-14 and $\sim 1.5 \pm$ $0.5 \mathrm{~km} \mathrm{~s}^{-1}$ for Clump-16, respectively. This line-width is a result of thermal width, internal turbulence, and possible infall. Since 

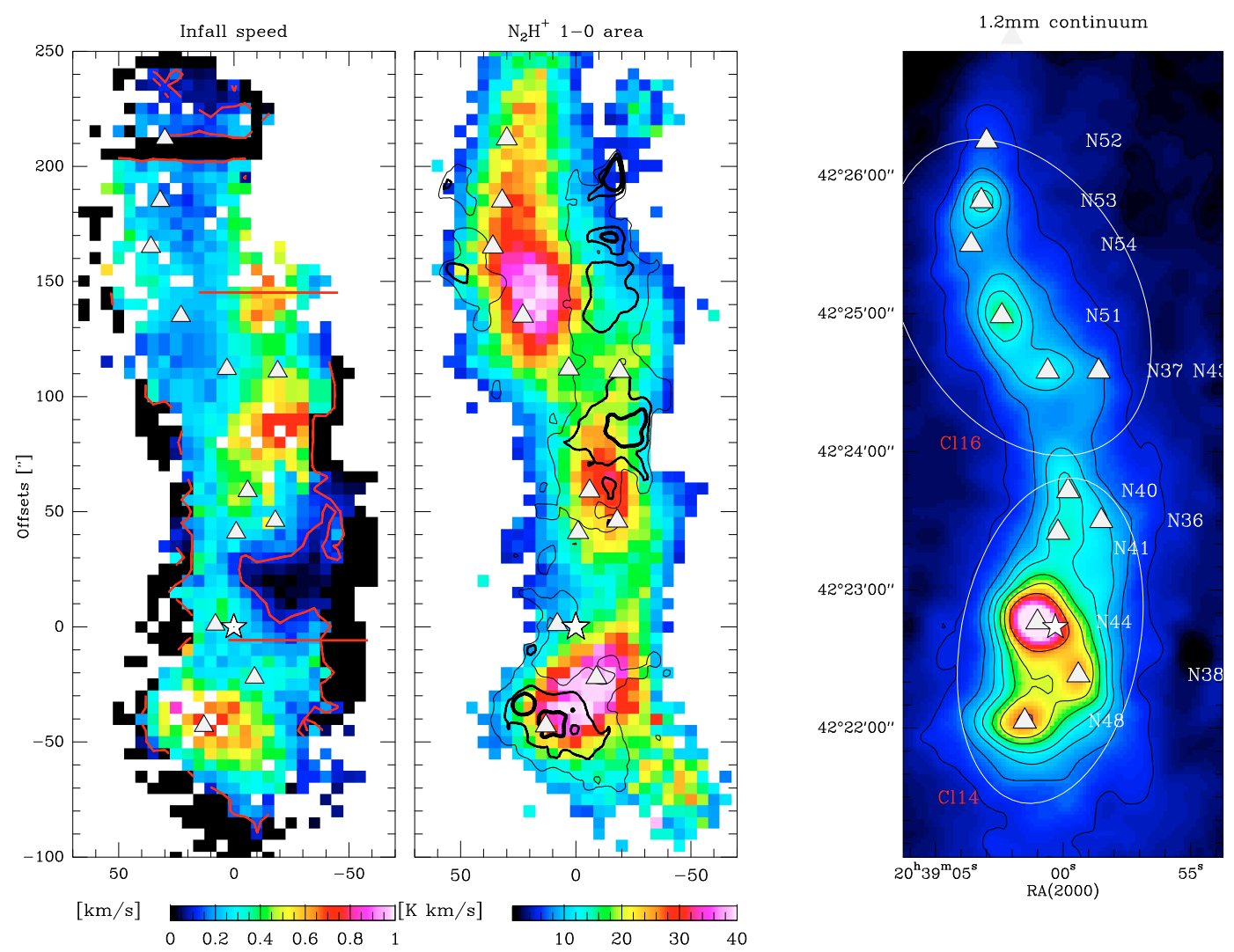

Fig. 15. Left: color plot of infall velocity. The red contour line indicates the thermal sound speed of $0.2 \mathrm{~km} \mathrm{~s}^{-1}$. Grey triangles mark mm-continuum sources. The two red lines indicate the cuts where we model spectra (Sect. 5.3). The cuts were selected to cross the center of each ellipse. Middle: contours of infall velocity (levels are $0.3,0.5,0.7 \mathrm{~km} \mathrm{~s}^{-1}$ ) overlaid on a plot of line-integrated $\mathrm{N}_{2} \mathrm{H}^{+}$intensity determined from a Gaussian line fit to the $-3 \mathrm{~km} \mathrm{~s}^{-1}$ component. It becomes obvious that the highest infall velocities are offset from density maxima. Right: mm-continuum emission map taken from Motte et al. (2007), where the two ellipses indicate the clumps considered for modeling and calculating infall properties. Note that all plots cover exactly the same area and have the same scales.

both turbulence and infall broaden the line, their parameter space must be explored carefully.

For the $\mathrm{HCO}^{+}$abundance, we take a value of $X\left(\mathrm{HCO}^{+}\right) 1-$ $5 \times 10^{-9}$ (e.g. Marseille et al. 2008) and adopt an isotope ratio ${ }^{12} \mathrm{C} /{ }^{13} \mathrm{C}=67$ (e.g. Lucas \& Liszt 1998). However, $\mathrm{HCO}^{+}$ is known to be depleted in cold environments and at densities higher than $\sim 10^{5} \mathrm{~cm}^{-3}$ (Tafalla et al. 2002). We expect this happens in Clump-16 but to a lesser extent in Clump-14 since this region contains a hot core. We thus introduce a threshold density $n_{\text {tresh }}$ in our model, with a sharp jump in the abundance profile if $n>\mathrm{n}_{\text {tresh }}$. The depletion factor is assumed to be in the range $1-100$.

Our strategy to constrain the infall speed was to vary the depletion ratio, the abundance, and the threshold density (between the given limits). To determine the best fit to the model, we performed a reduced $\chi^{2}$-test.

\subsubsection{Results}

Figures 16 and 17 show the observed and modeled spectra of $\mathrm{HCO}^{+}$and $\mathrm{H}^{13} \mathrm{CO}^{+}$for Clump-14 and Clump-16, respectively. For better orientation, the two cuts are indicated in Fig. 15. Table 2 summarizes the explored parameter space and gives the results for the best fitting model. We note that the spectra of both sources have broad wings caused by outflow emission that we did not account for in our modeling.
Table 2. Results of Simline modelling where $\alpha_{2}$ is the density profile exponent at the transition to the subthermal layer and $\mathrm{X}\left(\mathrm{HCO}^{+}\right.$the $\mathrm{HCO}^{+}$ abundance.

\begin{tabular}{lcc}
\hline \hline & Clump-14 & Clump-16 \\
\hline total mass & $4900 M_{\odot}$ & $3346 M_{\odot}$ \\
size & $0.52 \mathrm{pc}$ & $0.615 \mathrm{pc}$ \\
density profile $\left(\alpha_{2}\right)$ & -8.0 & -8.0 \\
mass-averaged $T$ & $20 \mathrm{~K}$ & $15 \mathrm{~K}$ \\
$\beta$ & -0.6 & -0.5 \\
line width & $2.5 \mathrm{~km} \mathrm{~s}^{-1}$ & $1.5 \mathrm{~km} \mathrm{~s}^{-1}$ \\
$X\left(\mathrm{HCO}^{+}\right)$ & $2.5 \times 10^{-9}$ & $2.5 \times 10^{-9}$ \\
${ }^{12} \mathrm{C} /{ }^{13} \mathrm{C}$ & 67 & 67 \\
depletion ratio & 7 & 28 \\
$\tau_{\mathrm{HCO}^{+}}$ & 25 & 26 \\
$\tau_{\mathrm{H}^{13} \mathrm{CO}^{+}}$ & 0.81 & 0.99 \\
$v_{\text {inf }}$ & $-0.6 \mathrm{~km} \mathrm{~s}^{-1}$ & $-0.5 \mathrm{~km} \mathrm{~s}^{-1}$ \\
\hline
\end{tabular}

\section{Clump-14}

For Clump-14, our best-fit model closely reproduces the spectral lines of $\mathrm{HCO}^{+}(\tau \sim 25)$ and $\mathrm{H}^{13} \mathrm{CO}^{+}(\tau \sim 0.8)$. It was necessary to use a rather large outer layer to reproduce well the absorption dip, which extends to $R \sim 0.9$ pc increasing by $20 \%$ the mass of our model. We found that a temperature profile of $\beta \sim-0.6$ and a mass-averaged temperature of $20 \mathrm{~K}$ provides a closest fit to our data, where the discrepancy in the peak intensity of the lines is smaller than $20 \%$. This also indicates that the inner part of this clump tends to be hotter, reaching up to $\sim 100 \mathrm{~K}$ in the central part. 


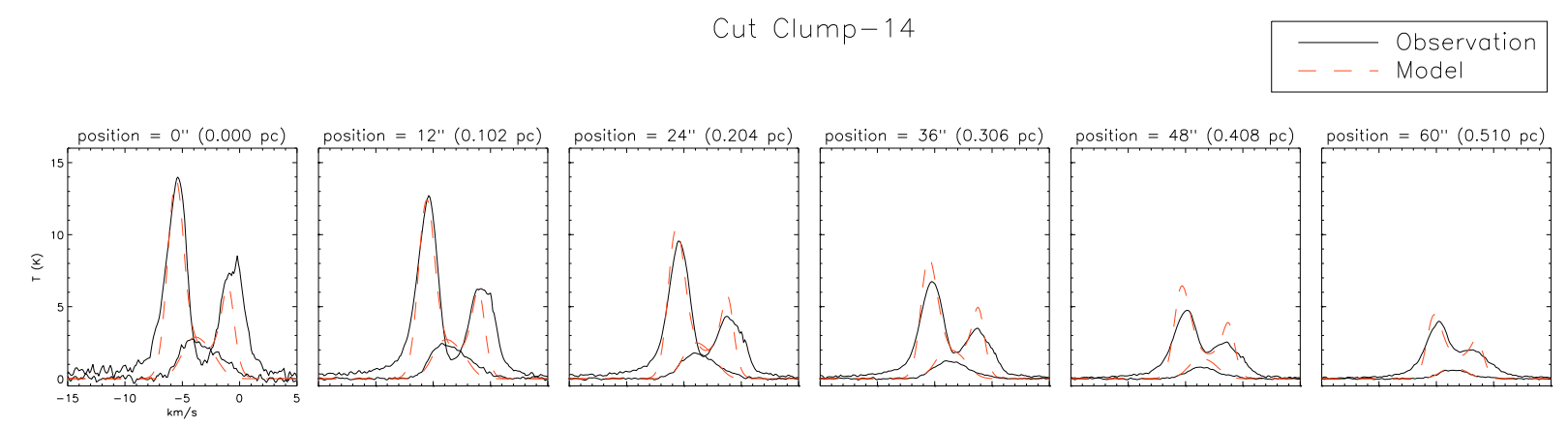

Fig. 16. Observed (black) and modeled (red) $\mathrm{HCO}^{+}$and $\mathrm{H}^{13} \mathrm{CO}^{+} 1 \rightarrow 0$ spectra of Clump-14 (see Fig. 15 for the location of the cut).

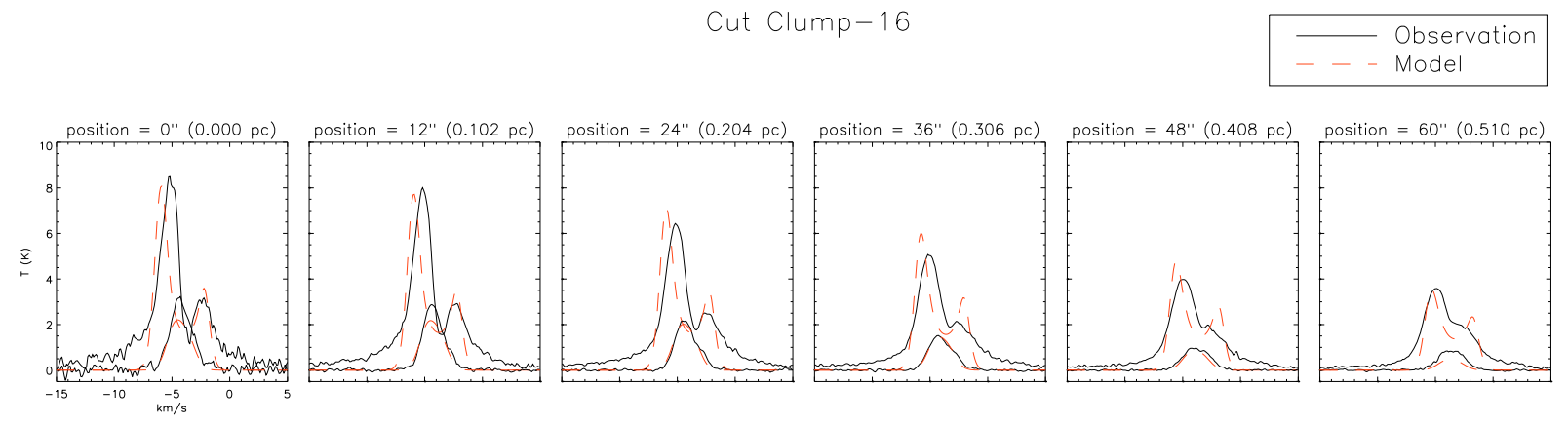

Fig. 17. The same like Fig. 16 but for Clump-16.

The depletion occurs at a radius of $0.25 \mathrm{pc}$ with a depletion ratio of $\sim 7$. This may be due to the temperature because the central region is a hot molecular core. With this configuration of geometry and parameters, we derive an infall speed of $\sim-0.6 \mathrm{~km} \mathrm{~s}^{-1}$, which compares well with the values derived from the Myers et al. method (see Sect. 5.2).

\section{Clump-16}

The best-fit model for Clump-16 (Fig. 17) reproduces very well the $\mathrm{H}^{13} \mathrm{CO}^{+}$line $(\tau \sim 1)$ but less well the optically thick $\mathrm{HCO}^{+}$ line $(\tau \sim 26)$. Since the densities are lower for this clump, the extended outer layer is smaller than that of Clump-14 and reaches out to $R_{\text {ext }} \sim 0.8 \mathrm{pc}$ reproducing the $10^{3} \mathrm{~cm}^{-3}$ density. As we kept the exponent $\beta$ for the temperature profile free, we found that $\beta=-0.5$ most closely fits our data. This results in a maximum of $T \sim 60 \mathrm{~K}$ in the central region. The depletion occurs at $R \sim 0.2 \mathrm{pc}$ with a depletion ratio of 28 , implying that in the central, very dense part of this clump, this molecule is strongly depleted.

The fit value of the infall speed is $\sim-0.5 \mathrm{~km} \mathrm{~s}^{-1}$, again within the range obtained from the more simple Myers et al. method. The strongest constraint on the infallvelocity is the ratio of the left-to-right wing intensity ratio, because the position of the plotted spectra is aligned with the optically thin line.

\subsection{Global infall properties of the DR21 filament}

Table 3 summarizes the infall properties of the two main infall regions that we defined in the Simline modeling. It is first important to understand that we discuss here the global dynamic features of the molecular clumps on a size scale of $\sim 0.5 \mathrm{pc}$, and not the physical properties of individual cores on a size scale of $\sim 0.1 \mathrm{pc}$.

The large global infall speeds on a $\sim 0.5 \mathrm{pc}$ size scale and the high masses of the clumps lead to large global infall rates of $\dot{M}$ of $5.8 \times 10^{-3} M_{\odot} \mathrm{yr}^{-1}$ and $2.8 \times 10^{-3} M_{\odot} \mathrm{yr}^{-1}$ for Clump-14
Table 3. Infall properties for Clump 14 and 16, i.e. the two main infall regions.

\begin{tabular}{lccccc}
\hline \hline & $\begin{array}{c}R^{a} \\
{[\mathrm{pc}]}\end{array}$ & $\begin{array}{c}M^{b} \\
{\left[M_{\odot}\right]}\end{array}$ & $\begin{array}{c}\left\langle n_{\mathrm{H}_{2}}\right\rangle^{c} \\
10^{5}\left[\mathrm{~cm}^{-3}\right]\end{array}$ & $\begin{array}{c}v_{\text {in }}{ }^{d} \\
{\left[\mathrm{~km} \mathrm{~s}^{-1}\right]}\end{array}$ & $\begin{array}{c}\mathrm{d} M / \mathrm{d} t^{e} \\
{\left[M_{\odot} \mathrm{yr}^{-1}\right]}\end{array}$ \\
\hline Clump 14 & 0.52 & 4900 & 1.26 & 0.6 & $5.8 \times 10^{-3}$ \\
Clump 16 & 0.615 & 3346 & 0.52 & 0.5 & $2.8 \times 10^{-3}$ \\
\hline
\end{tabular}

Notes. ${ }^{(a)}$ Equivalent radius of infall region. (b) Mass from mmcontinuum (see Table 2). ${ }^{(c)}$ Average $\mathrm{H}_{2}$ density, derived from the mass given in Col. 2. ${ }^{(d)}$ Infall speed, determined from Simline modelling (Sect. 5.3). ${ }^{(e)}$ Mass infall rate $\dot{M} \approx M / t=M v_{\text {in }} / R$.

and Clump-16, respectively. However, one has to distinguish between large-scale flow motions and local core collapse. The expression "infall", representing material that is falling onto a protostar or - more generally - into a gravitational potential well that could form many stars within a core that is gravitationally collapsing has to be used with care. In Fig. 15, we see that the highest values of "infall" speed do not always correspond to dense cores, only source N48 shows a local peak for $v_{\text {in. }}$ For the rest of the DR21 filament, the highest "infall" speeds are found in the less dense western part of the filament and not in the dense post-shock gas. In view of the high mass infall rates, this implies that there is a significant contribution of matter input either by directed flows (as would be the case for large-scale convergent flows) or gravitational collapse of the DR21 filament itself on a large scale. Both clumps have a low virial parameter $\alpha^{7}$ of 0.46 and 0.30 for Clump 14 and Clump 16, respectively, and are thus strongly gravitationally bound. Their freefall lifetimes $t_{\mathrm{ff}}{ }^{8}$ are $0.9 \times 10^{5} \mathrm{yr}\left(\right.$ Clump 14) and $1.3 \times 10^{5} \mathrm{yr}$

7 The virial parameter is defined to be $\alpha=M_{\mathrm{vir}} / M$ where the virial mass is $M_{\mathrm{vir}}\left[M_{\odot}\right]=699 \sigma^{2}\left[\mathrm{~km} \mathrm{~s}^{-1}\right]^{-2} R$ [pc] and the mass $M$ from Table 2.

${ }^{8} t_{\mathrm{ff}}[\mathrm{yr}]=1.37 \times 10^{6}\left(\frac{10^{3} \mathrm{~cm}^{-3}}{2 n\left(\mathrm{H}_{2}\right)}\right)^{0.5}$. 

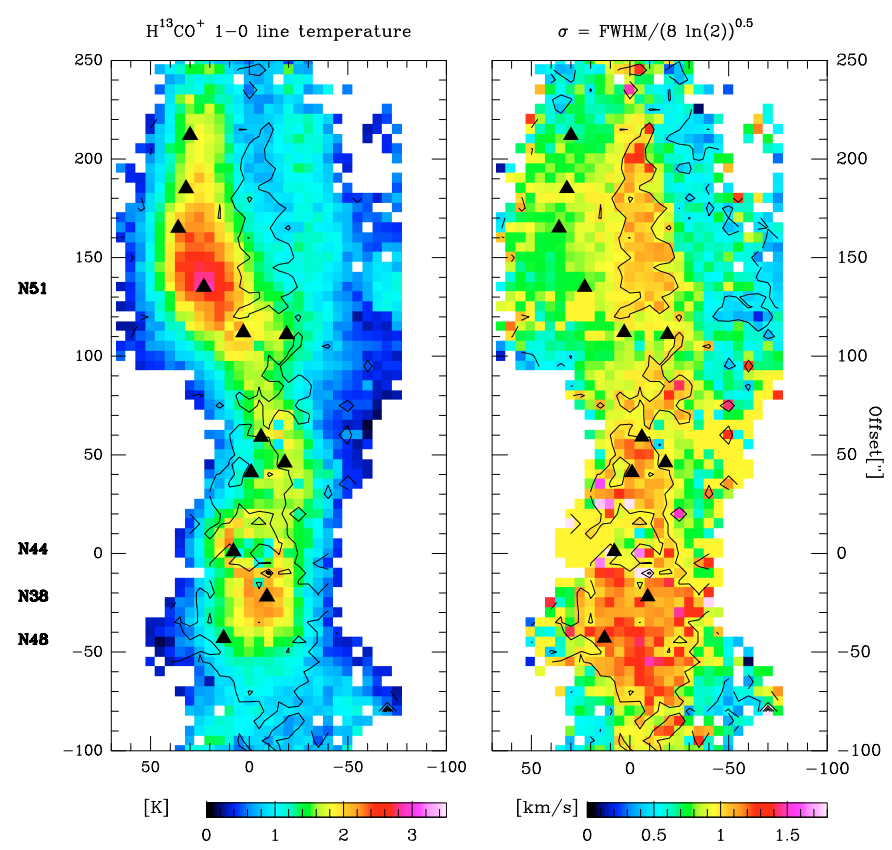

Fig. 18. Color scale maps of the results of Gaussian line fitting (temperature and velocity dispersion) to the $-3 \mathrm{~km} \mathrm{~s}^{-1}$ component of $\mathrm{H}^{13} \mathrm{CO}^{+}$ $1 \rightarrow 0$ along the DR21 filament (but excluding the region of DR21). Contours of velocity dispersion (second panel) at 0.75 and $1 \mathrm{~km} \mathrm{~s}^{-1}$ are overlaid on both maps.

(Clump 16), a factor 20-30 shorter than the sound crossing time, which indicates that they are indeed in global collapse (if the simple picture of a collapse onto a single gravitational potential is maintained). On the other hand, the infall rates are in agreement with the theoretical predictions of both the micro-turbulent core model (McKee \& Tan 2003) and the gravo-turbulent fragmentation model (e.g. Klessen et al. 1998, 2000, 2001; Padoan \& Nordlund 2002; Banerjee \& Pudritz 2007).

\subsection{The kinematic structure of the DR21 filament}

To further characterize the kinematic structure of the DR21 filament, we determine the line velocity and velocity dispersion using $\mathrm{H}^{13} \mathrm{CO}^{+}$and $\mathrm{N}_{2} \mathrm{H}^{+}$by fitting a Gaussian profile to the $3 \mathrm{~km} \mathrm{~s}^{-1}$ component as described in Sect. 5.2. For $\mathrm{N}_{2} \mathrm{H}^{+}$, we additionally fitted the hyperfine structure pattern of the molecule.

\subsubsection{Velocity dispersion}

Figure 18 reveals that the $\mathrm{H}^{13} \mathrm{CO}^{+}$main beam brightness temperature map shows a $\mathrm{V}$-shaped feature in the north of the filament. The western component is a factor 2-3 weaker than the eastern one, which has a pronounced peak at the position of N51. This peak is stronger than that towards N44/DR21OH(M) (the reverse is true for the line integrated intensity, see Fig. 5).

Most remarkable, however, is the velocity dispersion $\sigma$ (determined from the FWHM of the line with $\sigma=$ $F W H M /(8 \ln (2))^{0.5}$ ) seen in $\mathrm{H}^{13} \mathrm{CO}^{+}$as well as in $\mathrm{N}_{2} \mathrm{H}^{+}$(not shown here). The velocity dispersion increases towards the geometrical center of the filament from sonic levels to values of $\sim 1.3 \mathrm{~km} \mathrm{~s}^{-1}$. In the northern part of the filament (declination offset $>0^{\prime \prime}$ ), the gradient is stronger than in the southern part (south of N44/DR21OH-(M)) where we see a more homogeneous distribution of $\sigma$. Interestingly, in the northern part of the region highest $\sigma$-values are found between the two vertical features
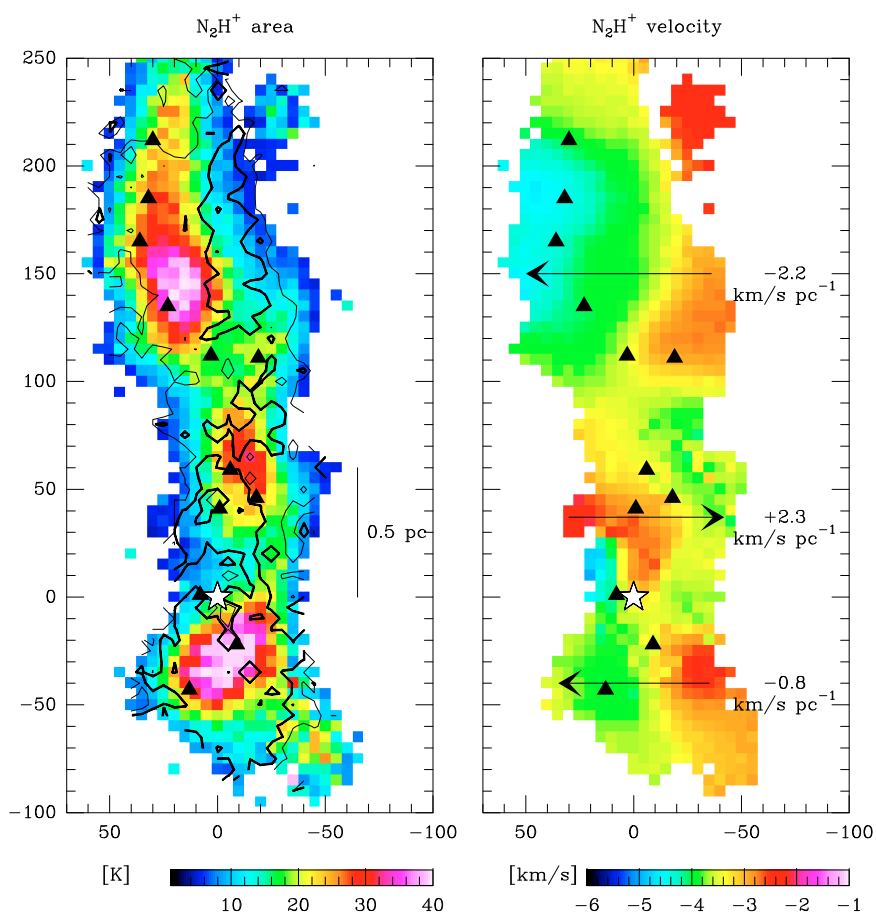

Fig. 19. Color scale maps of the results of Gaussian line fitting (line integrated intensity and line velocity) to the $-3 \mathrm{~km} \mathrm{~s}^{-1}$ component of $\mathrm{N}_{2} \mathrm{H}^{+} 1 \rightarrow 0$ along the DR21 filament (but excluding the region of DR21). Contours of velocity dispersion of $\mathrm{H}^{13} \mathrm{CO}^{+}\left(0.75\right.$ and $\left.1 \mathrm{~km} \mathrm{~s}^{-1}\right)$ are overlaid on the area map. Contour lines of the line integrated $(-1$ to $1 \mathrm{~km} \mathrm{~s}^{-1}$ ) emission of the $0 \mathrm{~km} \mathrm{~s}^{-1}$ component are overlaid on the velocity map (levels $1,2,3 \mathrm{~K} \mathrm{~km} \mathrm{~s}^{-1}$ ).

of $\mathrm{H}^{13} \mathrm{CO}^{+}$emission (with a decrease of $\sigma$ from $\sim 1 \mathrm{~km} \mathrm{~s}^{-1}$ to $\sim 0.8 \mathrm{~km} \mathrm{~s}^{-1}$ in the densest region in the east). This becomes even more obvious in Fig. 19 where we plot contours of $\sigma$ (from $\mathrm{H}^{13} \mathrm{CO}^{+}$) over a map of $\mathrm{N}_{2} \mathrm{H}^{+}$line integrated intensity (proportional to the column density). Thus, there is a clear offset between high $\sigma$ and high column densities. This scenario can be explained if it is assumed that clumps are produced by turbulent flows because in this case, the densest gas has been shocked and slowed down so that the largest velocity dispersions do not occur in the densest regions but in the outskirts (Klessen et al. 2005; Vazquez-Semadeni et al. 2008; Federrath et al. 2009). We return to this point in the discussion. The southern part (offset Dec $\left.<0^{\prime \prime}\right)$ seems to behave differently. The $\mathrm{N}_{2} \mathrm{H}^{+}$column density and $\mathrm{H}^{13} \mathrm{CO}^{+}$line temperature are highest in the core region ( $\sim 0.5$ pc diameter) of $\mathrm{N} 38$, while the velocity dispersion remains on a rather constant, high-level of around $1.2 \mathrm{~km} \mathrm{~s}^{-1}$ in an area of $\sim 1$ pc diameter. However, a projection effect could explain the difference: it is possible that a similar offset between velocity dispersion and highest density region is seen face-on.

\subsubsection{Velocity field}

Figure 19 shows the complex velocity field of the DR21 filament determined from $\mathrm{N}_{2} \mathrm{H}^{+}$(the $\mathrm{H}^{13} \mathrm{CO}^{+}$map not shown here looks very similar). Three main, basically horizontal, projected velocity gradients are observed. While the northern and southern part show a decrease of velocity from west to east $(-2.2$ and $-0.8 \mathrm{~km} \mathrm{~s}^{-1} \mathrm{pc}^{-1}$ ), the middle part has a positive value of $+2.3 \mathrm{~km} \mathrm{~s}^{-1} \mathrm{pc}^{-1}$. Interestingly, we observe in this region a "turnover" in the velocity gradient that corresponds to the material of the $0 \mathrm{~km} \mathrm{~s}^{-1}$ component "falling" onto the bulk of the 
filament (see Fig. 19). It is clear that the velocity pattern cannot be explained with a single rotation of the filament along a vertical axis. An alternative explanation would again be that at least a part of the observed motion is due to convergent flows (see Sect. 6) and/or rotation of the individual parts of the filament.

\section{Discussion}

The dynamic structure of the DR21 filament exhibits several remarkable features:

- several sub-filaments are connected to the DR21 filament, the most massive one, F3, being clearly linked to the DR21 $(\mathrm{OH})$ clump;

- global infall signatures are seen all across the filament (on a length scale of a few pc);

- the highest values of the velocity dispersion are found in a vertical projected region of low column density gas, offset from the dense clumps seen in $\mathrm{N}_{2} \mathrm{H}^{+}$;

- three horizontal (west-east) velocity gradients $(-0.8,+2.2$, $+2.3 \mathrm{~km} \mathrm{~s}^{-1} \mathrm{pc}^{-1}$ ) exist in the filament.

All of these findings point towards a very dynamic nature of the DR21 filament in which motions are not randomly distributed but show clear signs of systematic dynamics. These features all point to local converging flows being the driving source of the whole filament, with an increase in density at the stagnation points of the flows (Sect. 6.1). In addition, the large-scale infall signatures observed all over the filament point towards a scenario in which gravity is the dominant driving force on the scale of the filament (Sects. 6.2 and 6.3). On the other hand, the origin of the large-scale flows is difficult to constrain. It is possible that the large-scale dynamics are a remains of the formation process of the molecular complex, but may also originate either from self-gravity on larger scales, or large-scale turbulence injection (Sects. 6.4 and 6.5).

\subsection{Organized motions: signposts of convergent flows}

The clouds of the whole Cygnus North region are very similar in their physical properties (morphology, density etc.) to clouds that were created by colliding flow scenarios in models (e.g. the final stage of the Gf2 model from Heitsch \& Hartmann 2008). The DR21 filament is part of a complex network of interconnected filamentary structures (Sect. 4.1) and could thus indeed be the result of a convergent, turbulent flow. In particular, the offset of the infall speed from the densest regions is consistent with a scenario where supersonic flows shock at their stagnation points are offset from the dense postshock gas on both sides of the filament. In this case, high-density clumps/cores are built up at the stagnation points of the colliding flow and would explain the distribution of the dense mm-cores along the DR21 filament. It suggests that the cores are dynamically distributed through the region before they evolve into intermediate or high-mass starforming clusters. From the mm-continuum survey of Motte et al. (2007), we determined that approximately $15 \%$ of the mass of the filament is contained in dense cores, in accordance with the predictions of Heitsch \& Hartmann (2008).

Another observational results that agrees with supersonic isothermal simulations is the velocity field and the distribution of the velocity dispersion $(\sigma)$. We observe different velocity gradients along the filament. Figure 19 shows in the northern and southern part of the filament a negative gradient in the WEdirection whereas at the position of DR21(OH) (where the subfilament falls onto the densest region), this gradient is reversed. It is unlikely that the gradients are only caused by rotation (see, e.g. Goodman et al. 1993, for typical observational signatures of uniform rotation). Moreover, they can be explained in the turbulent colliding flow view, where clumps are sheared and compressed due to the flow motions and retain the signature of the external flow that formed them (Ballesteros-Paredes et al. 1999; Vazquez-Semadeni et al. 2008) so that only parts of the filaments are rotating.

The shock front created by the colliding flows creates an inner dense post-shock region and an outer lower-density region of supersonic inflow. Cores are then formed by "gravoturbulent fragmentation" (Klessen et al. 2005; Hennebelle \& Chabrier 2008), which characterizes a hierarchy of smaller and smaller entities. The local collapse of a core occurs when self-gravity overcomes the gas pressure (including the turbulent contribution to pressure). The whole process leads to a higher velocity dispersion outside the clump/core than in the more quiescent post-shocked interior (Klessen et al. 2005; Gomez et al. 2007; Vazquez-Semadeni et al. 2008), which is precisely what we observe in the DR21 filament. Figure 1 in Klessen et al. (2005) illustrates this scenario in a non-magnetic SPH (smoothed particle hydrodynamics) model. Here, we provide evidence of the same scenario in Fig. 20, where we show a map of the velocity dispersion obtained in a driven turbulence simulation by Federrath et al. (2009). The simulation used fully compressive (curl-free) forcing to excite turbulent motions, though models with solenodial (divergence-free) forcing (not shown here) produce similar results. In general, compressive forcing produces greater density contrasts with higher density enhancements and larger voids than solenodial forcing (Federrath et al. 2008, 2009). This could be the case for the DR21 filament since it is here where we observe the massive dense cores with densities higher than $10^{5} \mathrm{~cm}^{-3}$ and masses up to $200 M_{\odot}$. The velocity field primarily consists of converging flows, which create strong density enhancements. Column density contours are overlaid on the velocity dispersion, showing that $\sigma$ and density peaks are offset from one another, as in our observations shown in Fig. 15 (middle panel) of the DR21 filament. High density gas is primarily found in regions of low $\sigma$, while lower density regions have higher $\sigma$. We emphasise that the simulation did not include self-gravity. Nevertheless, the velocity dispersion-density structure is similar to what is observed in the DR21 filament, which indicates that the observed structures can be produced by converging supersonic gas flows only, without the necessity of a global gravitational collapse. However, converging gas flows are also created by global collapse. If supersonic flows and global collapse act simultaneously, it is difficult to disentangle their individual contribution to the creation of the velocity dispersion-density structure seen in our observations and in the simulations (see Sect. 6.5 below for a general discussion of the origin of converging flows).

\subsection{Self-gravity, the main driving force in the DR21 filament?}

Observationally, the ubiquity of the blue-shifted asymmetric line profiles in all optically thick lines indicates inflow motions. This is a strong hint that self-gravitation plays a significant role for the dense and massive DR21 filament. In addition, the most massive sub-filament F3, which is a good candidate to flow towards the main filament, seems to bend towards the center of the largest gravitation well of the whole filament, the DR21(OH) clump (see Sect. 5.1). This bend may be indicative of a dominant role of 


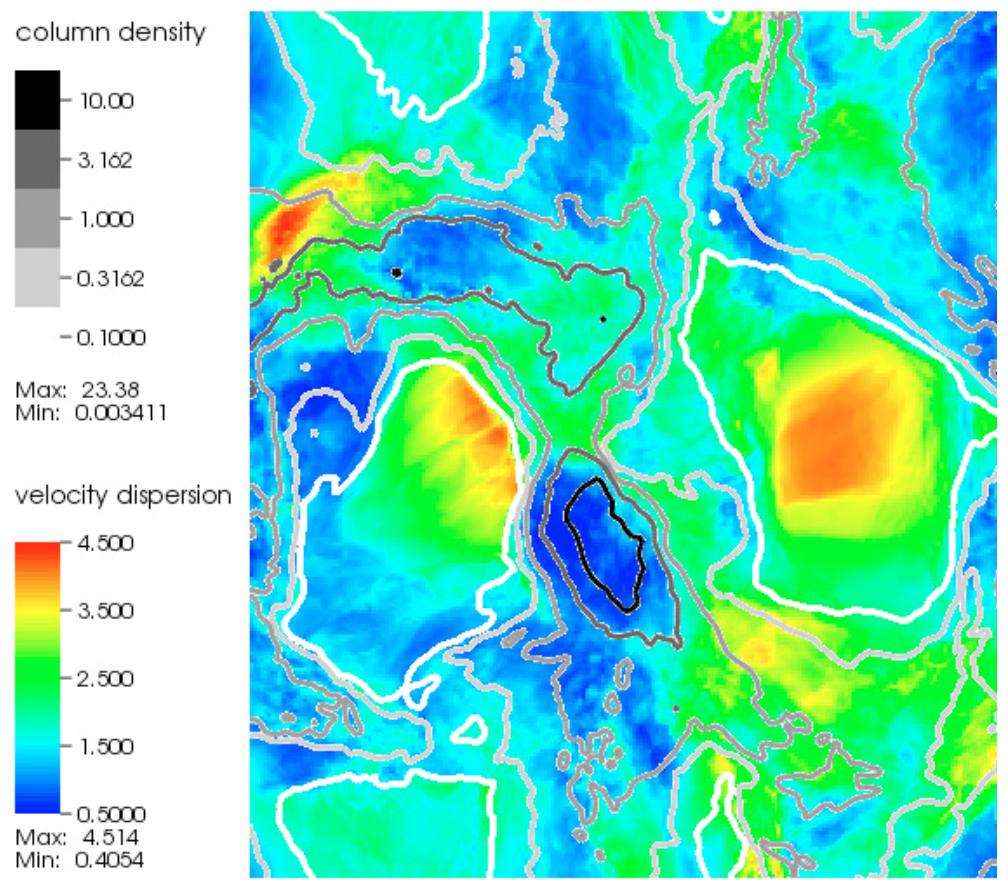

Fig. 20. Results of a hydrodynamic turbulence simulation by Federrath et al. (2009), considering only compressive (curl-free) forcing. The Mach numbers reached in this simulation are comparable to the typical Mach numbers observed in the DR21 filament. The color-coded velocity dispersion is in units of the sound speed, while contours of column density are overlaid on it. The simulation was performed with the grid code FLASH3 and used $1024^{3}$ computational elements. This figure can be directly compared to Fig. 15 (middle panel), also showing a prominent offset between the maxima of velocities and column densities.

gravity as the flowing material approaches the main filament. At larger distances, the effect of large-scale magnetic fields could be the cause that keeps it in an almost east-west direction (see Sect. 6.3).

As shown in Sect. 6.1, we are able to explain the spatial displacement between the maximum of the velocity dispersion and the dense gas and the velocity field of the DR21 filament in the context of supersonic isothermal simulations that do not include self-gravity. However, models of isothermal, non-magnetic, but self-gravitating turbulence produce a velocity divergence of typically $0.6 \mathrm{~km} \mathrm{~s}^{-1} \mathrm{pc}^{-1}$ for a $1 \mathrm{pc}$ region (Vazquez-Semadeni et al. 2008). The authors propose that the assumption that the velocity dispersion is produced only by random motion is incomplete and that even in the presence of driven turbulence a part of the observed velocity dispersion is caused by clump-scale inward motions due to gravitational collapse. In this large-scale inflow (LSI) scenario, the velocity field on all scales includes a significant inflow component.

\subsection{Role of magnetic field: MHD with self-gravity modeling}

We observed several sub-filaments that are attached to the DR21 filament (Sects. 4.2). The orientation of the sub-filaments is not arbitrary: they follow the magnetic field lines that run orthogonal to the NS-orientated DR21 filament (Vallée \& Fiege 2006; Kirby 2009). Magnetohydrodynamic turbulence models precisely produce such filamentary structures (Fig. 21) that are aligned with the magnetic field (Fig. 22). Hennebelle \& Audit (2008) found that self-gravitating MHD models produce more filamentary structures than purely hydrodynamic models. These numerical simulations were specifically designed for this object and the initial conditions resemble those used in Peretto et al. (2007). They consist of an elongated clump with an initial aspect ratio of 2 and a density profile $\rho(r, z)=\rho_{0} /\left(1+\left(r / r_{0}\right)^{2}+\left(z / z_{0}\right)^{2}\right)$, where $\left.r=\sqrt{(} x^{2}+y^{2}\right), z_{0}=2 r_{0}, r_{0}=5 \mathrm{pc}$ and $\rho_{0}=500 \mathrm{~cm}^{-3}$.
The density at the edge of the cloud is equal to $50 \mathrm{~cm}^{-3}$ and is 10 times lower than the value outside the cloud. Turbulence is seeded initially in such a way that the clump is approximately in virial equilibrium. The initial temperature is $10 \mathrm{~K}$ and the clump is threaded by a magnetic field parallel to the $x$-axis. Its intensity is proportional to the cloud column density and the peak value is about $7 \mu \mathrm{G}$ making the magnetic energy about 4 times lower than the turbulent one and 5 times higher than the thermal energy. This simple initial configuration for the magnetic field is suggested by the observations of Vallée \& Fiege (2006), who show that the magnetic field is indeed perpendicular to the major axis of DR21. The simulation was performed with the Ramses code (Teyssier 2002, Fromang et al. 2006). Since the cloud occupies a small fraction (about 5 percent) of the computational domain, the following strategy is adopted. Initially a uniform $128^{3}$ computational grid is used (level 7). Then, all the cells of density above $10 \mathrm{~cm}^{-3}$ and $40 \mathrm{~cm}^{-3}$ are refined to the level 8 and 9 , respectively. This ensures that initially the clump is described with about $3 \times 10^{6}$ computing cells. As the collapse proceeds, the resolution is adjusted requiring at least 10 cells per Jeans length up to level 14.

In the left-hand panel of Fig. 21, the distribution of the column density is shown and in the right-hand figure panel, the velocity field vectors are overlaid on top. The column density is smoothed to a beam of $\sim 0.1 \mathrm{pc}$ angular resolution (that of the IRAM $30 \mathrm{~m}$ molecular line observations). The resemblance to the DR21 filament is evident with an elongated vertical main filament and several subfilaments attached to it. Several clumps/cores (size scales $<0.5 \mathrm{pc}$ ) with high $\mathrm{H}_{2}$ column densities $\left(>10^{24} \mathrm{~cm}^{-2}\right)$ have formed in the filament, in agreement with our observations.

Figure 22 shows the mean velocity dispersion, the mean velocity field along the line of sight, and the averaged magnetic field. For the velocity dispersion $\sigma$, we observe the same offset between regions of high density and $\sigma$ as seen in Fig. 20. 

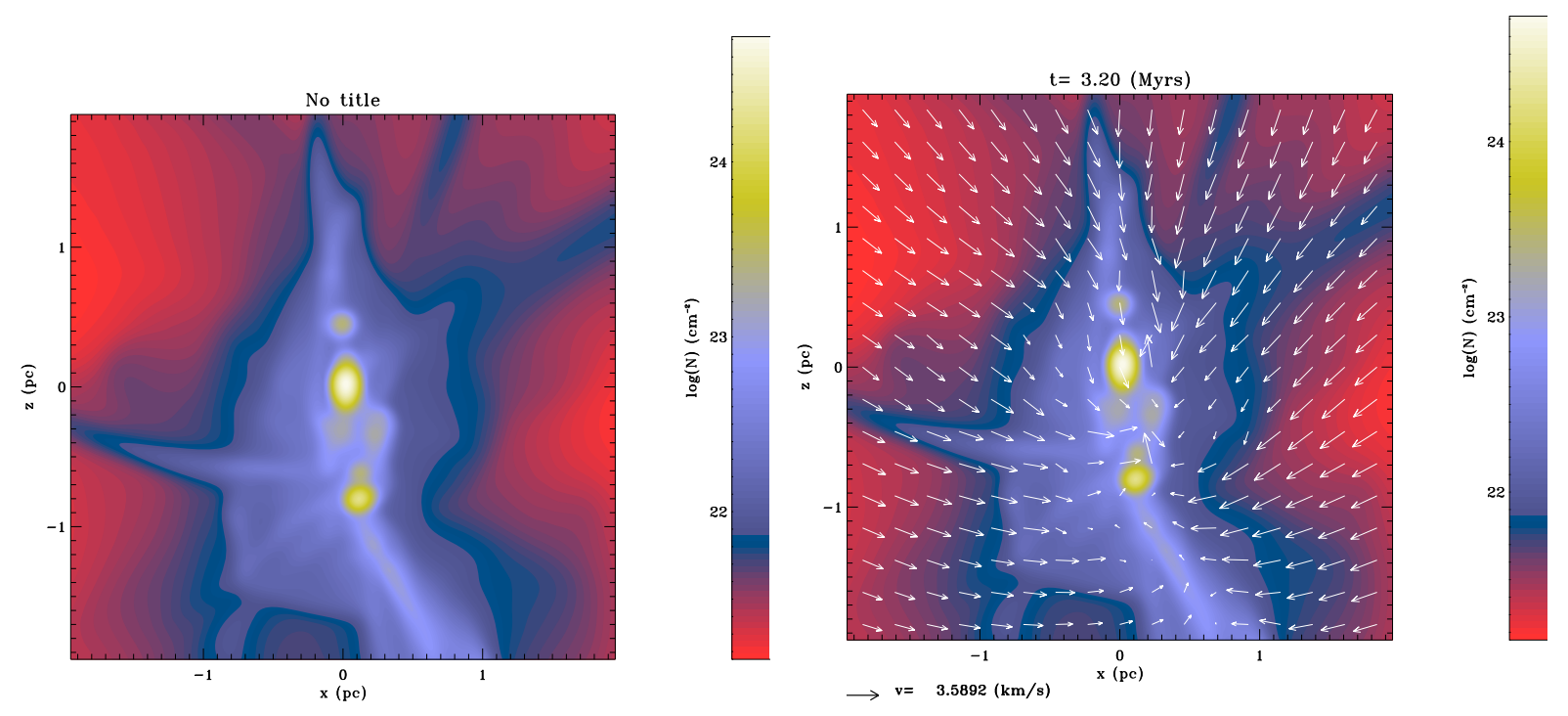

Fig. 21. In the left-hand plot, we show the column density distribution from an MHD code (Hennebelle et al.; Teyssier 2002; Fromang et al. 2006). In the right plot, the projected velocity field is overlaid on the column density distribution.

The modeled velocity field is as inhomogenous as the observed one, though the gradients have slightly larger values (up to a few $\mathrm{km} \mathrm{s}^{-1} \mathrm{pc}^{-1}$ ) and the same direction (no turn in sign as we found for the region around the DR21(OH) clump). The magnetic field is particularly interesting. The total magnetic intensity is displayed in Fig. 22 (bottom), the arrows showing the direction of the magnetic field in the $x z$ plane. The sub-filaments are clearly aligned along the magnetic field and even sometimes pinched by it. This is in full accordance with our observations and points toward a scenario in which the DR2 1 filament is fed with material along EW-orientated magnetic field lines. A full network of these subfilaments was detected in recent JCMT ${ }^{12} \mathrm{CO} 3 \rightarrow 2$ data (Richer et al., in prep.). A more detailed discussion of these observations and MHD modelling will be presented in a forthcoming paper (Csengeri, Hennebelle et al., in prep.).

However, the prinicipal driver of these flows remains unclear. Is it external, large-scale driven turbulence or the gravitational attraction of the gas itself, or both? A possible scenario is that first, the turbulence creates the density fluctuations (clumps and filaments) by means of locally convergent flows, and then, for some of the more massive fluctuations, gravity takes over, making the inflow stronger and driving the material to higher densities. We probably see both effects in the DR21 filament: turbulent compression and self-gravity. The northern part is probably in an earlier stage where the external flow is still important in shaping the velocity field (hence the offset of the 1st and 2nd velocity moments from the density map). In the southern part, we see a more advanced stage in which gravity dominates and gravitational infall extends all the way to the center (leading to the coincidence of the density and velocity structures).

\subsection{Are the observed sub-filaments able to maintain the formation of the DR21 filament?}

We observed that several sub-filaments are attached to the DR21 filament (Sect. 4.2), possibly serving as a reservoir of gas for further mass growth. These organized flows help to sustain high accretion rates for times long enough to build up very massive clumps/cores (Vazquez-Semadeni et al. 2009). We note that the DR21(OH)-clump has a mass of higher than $5000 M_{\odot}$ in a radius of $\sim 0.5 \mathrm{pc}$, which makes it one of the most massive clumps known in the Galaxy. The F3-filament (Sect. 5.1) has an average mass input rate of $1.9 \times 10^{-3} M_{\odot} \mathrm{yr}^{-1}$ so that it may contribute up to $\sim 30 \%$ of the total mass infall rate of $5.8 \times 10^{-3} M_{\odot} \mathrm{yr}^{-1}$ observed for the DR21(OH) clump as a whole (Clump 14, see Table 3) for the next $2.1 \times 10^{6} \mathrm{yr}$. Accounting for the other less massive sub-filaments, which may also contribute, this indicates that the mass flow rates are of the correct order of magnitude to explain the formation of the DR21 filament. On the other hand, they seem insufficient to build the present $5000 M_{\odot} \mathrm{DR} 21(\mathrm{OH})$ clump, which suggests that the mass flow rate must have been higher in the past and that the process that produced the DR21 filament may be declining.

\subsection{Origin of the converging flows that formed the DR21 filament}

One physical scenario that intrinsically includes strong dynamics is that of molecular clouds and complexes being formed by large-scale HI convergent flows, which would then drive the whole dynamics of the complex down to the star-formation scale (Klessen et al. 1998; Folini \& Walder 2006; Vazquez-Semadeni et al. 2007; Heitsch et al. 2008; Banerjee et al. 2009; Federrath et al. 2009). Common for all "convergent-flow" scenarios is the basic principle that the clouds result from a pileup of material, i.e. atomic hydrogen, from large-scale supersonic flows, caused by energetic events such as supernovae, expanding HIIregions, galactic spiral density waves. The driving force could be the injection of supersonic turbulence on large scales in a pre-existing molecular complex. Supersonic turbulence necessarily leads to organized supersonic motions on smaller scales, and then leads to the formation of filaments and clumps, and then stars. Finally, the self-gravity of a pre-existing molecular complex may play a role in driving the flows. Altogether, it is difficult to trace back the origin of the large-scale motions observed here. But at least on the observed scales, the motions are supersonic and are converging to form dense structures such as the DR21 filament. Whether a more quasi-static evolution is possible on smaller scales or in other parts of the complex to drive the evolution towards (high-mass) star formation cannot be addressed by the present observations. In Csengeri et al. (2010), we address the kinematic state of the small scale, densest structures 

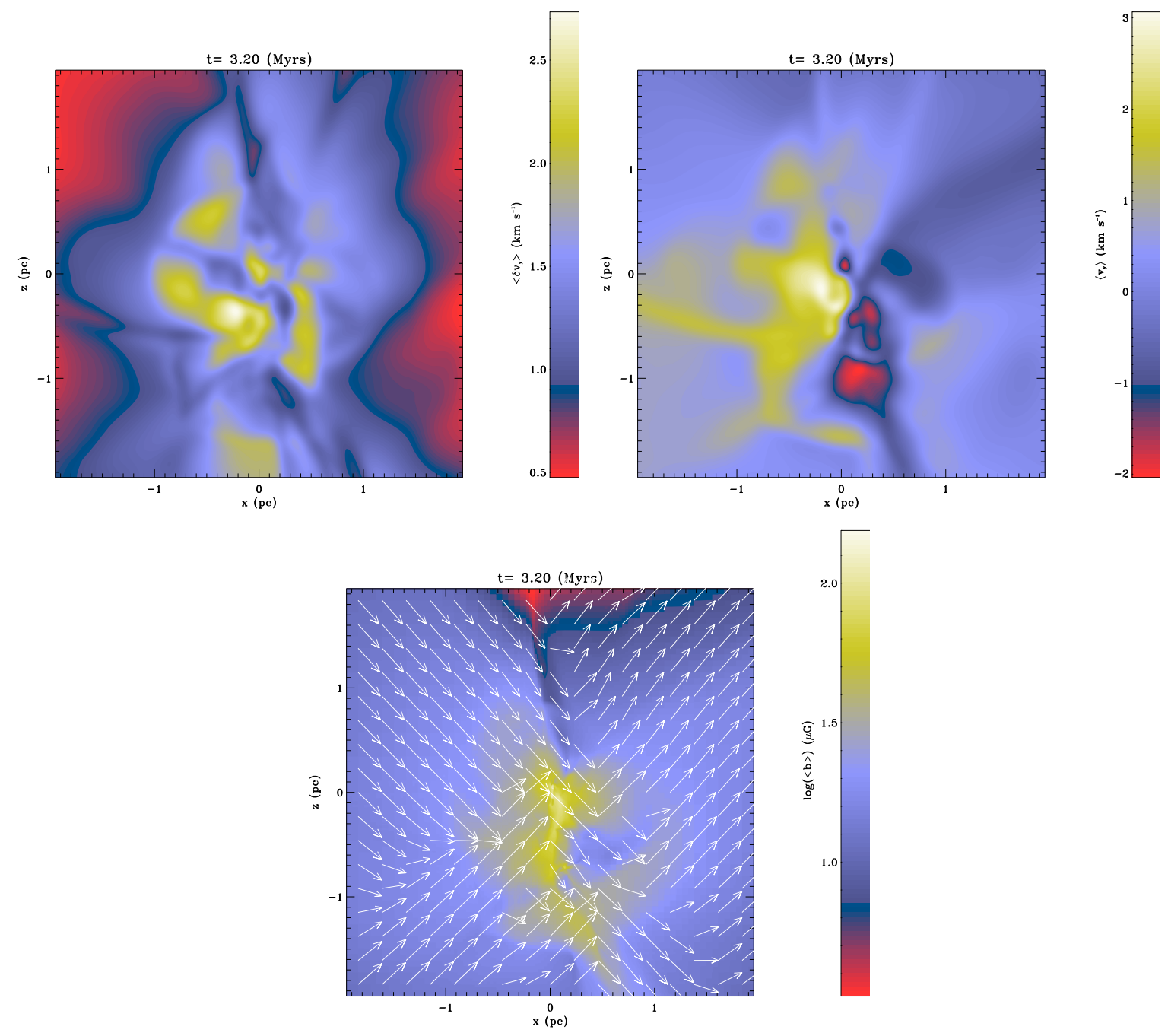

Fig. 22. Results from the same MHD turbulence model (Hennebelle et al.; Teyssier 2002; Fromang et al. 2006) shown in Fig. 20. Top: velocity dispersion (left) and velocity field (right). Bottom: magnetic field with field vectors overlaid on it.

in the complex, the i.e., massive dense cores, using PdBI data (Csengeri et al., in prep).

To distinguish the formation scenarios more clearly on large scales, it is not clear which other observational signatures can be expected. The DR21 filament itself is already a rather dense structure and in order to detect residues of the formation process, it is neccessary to inspect the distribution of low-density, subsonic gas using atomic hydrogen and low-density tracers such as ${ }^{12} \mathrm{CO}$. We will address this point in a subsequent paper (Csengeri, Hennebelle et al., in prep.).

\section{Summary and conclusions}

We have presented a detailed molecular line study of the molecular ridge containing the star-forming regions DR21 and DR21 $(\mathrm{OH})$. This ridge is embedded in a large-scale network of filamentary structures, revealed by our maps of ${ }^{13} \mathrm{CO} 1 \rightarrow 0$, CS $2 \rightarrow 1$, and $\mathrm{N}_{2} \mathrm{H}^{+} 1 \rightarrow 0$ emission obtained with the FCRAO. It is the most massive (around $30000 M_{\odot}$ ) and dense (average density $\sim 10^{4} \mathrm{~cm}^{-3}$ ) filament within the region and is labeled by us the "DR21 filament". Several sub-filaments are linked to the DR21 filament, the most massive one runs orthogonal to the North-South oriented ridge and has a mass (determined from ${ }^{13} \mathrm{CO} 1 \rightarrow 0$ ) of $2600 M_{\odot}$ and an average density of $690 \mathrm{~cm}^{-3}$. Its inferred dynamical time is $\sim 2 \times 10^{6} \mathrm{yr}$.
Higher angular resolution IRAM molecular line observations in $\mathrm{HCO}^{+}, \mathrm{H}^{13} \mathrm{CO}^{+},{ }^{12} \mathrm{CO} /{ }^{13} \mathrm{CO} 2 \rightarrow 1, \mathrm{C}^{34} \mathrm{~S}, \mathrm{~N}_{2} \mathrm{H}^{+}$, and $\mathrm{H}_{2} \mathrm{CO}$ resolve the detailed structure of the DR21 filalment. The $\mathrm{H}^{13} \mathrm{CO}^{+} 1 \rightarrow 0$ data show how the sub-filament seen in ${ }^{13} \mathrm{CO}$ connects directly to the DR21(OH) clump. From the ${ }^{12} \mathrm{CO} 2 \rightarrow 1$ line mapping, we confirmed the known outflow sources DR21 and DR21(OH) and detected three new ones, correlated with the mm-continuum sources N53, N44, and N45 (Motte et al. 2007). The $\mathrm{HCO}^{+} 1 \rightarrow 0$ line shows self-absorbed lines across the whole filament. Since optically thin lines peak in the gap and the blue wing of $\mathrm{HCO}^{+}$is more intense than the red one, we conclude that this emission feature is due to infalling gas. The typical infall speed, determined with a simple method described in Myers et al. (1996), is $0.6-0.8 \mathrm{~km} \mathrm{~s}^{-1}$. A more sophisicated non-LTE modelling of the $\mathrm{HCO}^{+}$and $\mathrm{H}^{13} \mathrm{CO}^{+}$lines using the Simline radiative transfer code yields an infall speed of 0.5 and $0.6 \mathrm{~km} \mathrm{~s}^{-1}$ for the northern and southern part of the DR21 filament (but excluding the DR21 region itself), respectively.

The kinematic structure of the DR21 filament is remarkable. We measured, using the $\mathrm{N}_{2} \mathrm{H}^{+}, \mathrm{H}^{13} \mathrm{CO}^{+}$, and $\mathrm{HCO}^{+}$maps, the highest values of the velocity dispersion in a vertical column of low column density gas that is offset from the dense clumps seen in $\mathrm{N}_{2} \mathrm{H}^{+}$. These results can be explained if the filament was produced by turbulent flows. In this case, the densest gas has been shocked and slowed down so that the largest velocity 
dispersions do occur not in the densest regions but in the outskirts (Klessen et al. 2005; Vazquez-Semadeni et al. 2008). We also observe three horizontal (west-east) velocity gradients $\left(-0.8,-2.2,+2.3 \mathrm{~km} \mathrm{~s}^{-1} \mathrm{pc}^{-1}\right)$ in the filament in which the one with the positive value marks the location where the filament is "falling" onto the DR21(OH) clump. This velocity pattern cannot be explained by a single rotation of the filament along a vertical axis. An alternative explanation would be that at least part of the observed motions is due to convergent flows.

By comparing our observations of the DR21 filament with a hydrodynamic (Federrath et al. 2009) and a magnetohydrodynamic turbulence model (Hennebelle et al. 2008; Teyssier 2002; Fromang et al. 2006), we infer that a very dynamic and fast mode of star formation occurs in the filament, in which gas is continuously replenished by subfilaments attached to the main filament. These subfilaments are aligned with the magnetic field direction that is perpendicular to the DR21 filament (Vallee \& Fiege 2006). The DR21 filament is globally collapsing. All our observational findings are incompatible with the view of a quasi-static, pressure-bounded clump scenario.

Acknowledgements. We thank A. Belloche and D. Poelman for useful discussions on line radiative transfer modelling.

A part of this work was supported by the French Agence National de la Recherche (ANR) project PROBeS No. 08-blanc-0241. T. Csengeri acknowledges support from the FP6 Marie-Curie Research Training Network "Constellation: the origin of stellar masses" (MRTN-CT-2006-035890).

R.S.K. acknowledges financial support from the German Bundesministerium für Bildung und Forschung via the ASTRONET project STAR FORMAT (grant 05A09VHA) and from the Deutsche Forschungsgemeinschaft (DFG) under grants Nos. KL 1358/1, KL 1358/4, KL 1359/5, KL 1358/10, and KL 1358/11. R.S.K. furthermore thanks for subsidies from a Frontier grant of Heidelberg University sponsored by the German Excellence Initiative and for support from the Landesstiftung Baden-Württemberg via their program International Collaboration II (grant P-LS-SPII/18). R.S.K. also thanks the KIPAC at Stanford University and the Department of Astronomy and Astrophysics at the University of California at Santa Cruz for their warm hospitality during a sabbatical stay in spring 2010 .

\section{Appendix A: Calculation of physical parameters}

\section{A.1. Abundances}

We use a standard $\left[{ }^{12} \mathrm{CO}\right] /\left[{ }^{13} \mathrm{CO}\right]$ ratio of 67 (Lucas et al. 1998).

The $\left[\mathrm{HCO}^{+}\right] /\left[\mathrm{H}_{2}\right]$ abundance in high-mass star-forming regions is typically quoted to be $1.5-2.5 \times 10^{-9}$ (Peretto et al. 2006; Marseille et al. 2008). For our mass determination from $\mathrm{H}^{13} \mathrm{CO}^{+}$, we apply the abundance value obtained from the Simline modelling (Sect. 5.3), which is $2.5 \times 10^{-9}$.

\section{A.2. Determination of mass from molecular lines}

The total column density of any optically thin molecule can be determined from

$N\left[\mathrm{~cm}^{-2}\right]=f\left(T_{\mathrm{ex}}\right) \int T_{\mathrm{mb}}[\mathrm{K}] \mathrm{d} v\left[\mathrm{~km} \mathrm{~s}^{-1}\right]$

where

$f\left(T_{\mathrm{ex}}\right)=\frac{3 h Z}{8 \pi^{3} \mu^{2} J_{\mathrm{t}}} \frac{\exp \left(h v / k T_{\mathrm{ex}}\right)}{\left[1-\exp \left(-h v / k T_{\mathrm{ex}}\right)\right]\left(J\left(T_{\mathrm{ex}}\right)-J\left(T_{\mathrm{BG}}\right)\right)}$

and the Partition function is given by

$Z=\frac{2 k T_{\mathrm{ex}}}{h v}+1 / 3$

in which $h$ and $k$ denote the Planck and the Boltzman constants, respectively, $v$ is the frequency $[\mathrm{GHz}], \mu$ is the dipole moment
[Debye], $J_{\mathrm{t}}$ is the upper value of the rotational quantum number and $\int T_{\mathrm{mb}} \mathrm{d} v$ is the velocity integrated line intensity on a main beam temperature scale

$$
J\left(T_{\mathrm{ex}}\right)=\frac{h v}{k\left(\exp \left(h v /\left(k T_{\mathrm{ex}}\right)-1\right)\right.}
$$

and $J\left(T_{B G}\right)=J(2.7 K)$.

The values for the $\mathrm{H}^{13} \mathrm{CO}^{+} 1 \rightarrow 0$ transition are $v=$ $86.75433 \mathrm{GHz}, h v / k=4.163 \mathrm{~K}, \mu=3.3$ Debye, $J_{\mathrm{t}}=1$, and $J(2.7 \mathrm{~K})=1.13$. The temperature-dependent factor $f\left(T_{\mathrm{ex}}\right)$ is $(2.43,2.84,4.39,9.4) \times 10^{12}$ for $10,20,40$, and $100 \mathrm{~K}$, respectively.

The values for the ${ }^{13} \mathrm{CO} 1 \rightarrow 0$ transition are $v=$ $110.201 \mathrm{GHz}, h v / k=5.29 \mathrm{~K}, \mu=0.112$ Debye, $J_{\mathrm{t}}=1$, and $J(2.7 \mathrm{~K})=0.868$. The temperature-dependent factor $f\left(T_{\mathrm{ex}}\right)$ is $(0.91,1.25,1.66,2.08) \times 10^{15}$ for $10,20,30$, and $40 \mathrm{~K}$, respectively.

The $\mathrm{H}_{2}$ column density from $\mathrm{H}^{13} \mathrm{CO}^{+}$is calculated to be

$N\left(\mathrm{H}_{2}\right)\left[\mathrm{cm}^{-2}\right]=2.43 \times 10^{10} N\left(\mathrm{H}^{13} \mathrm{CO}^{+}\right)$

and for ${ }^{13} \mathrm{CO} 1 \rightarrow 0$ from

$N\left(\mathrm{H}_{2}\right)\left[\mathrm{cm}^{-2}\right]=4.7 \times 10^{5} N\left({ }^{13} \mathrm{CO}\right)$.

The final mass is determined to be

$M\left[M_{\odot}\right]=N\left(\mathrm{H}_{2}\right) d^{2} A(\pi / 180)^{2} 2 m_{\mathrm{H}} \mu$,

where the projected area $A$ is in square degrees, the distance $d$ in parsec, the mass of hydrogen $m_{\mathrm{H}}$, and $\mu=1.36$.

\section{References}

Adler, D. S., Lo, K. Y., Wright, M. C. H., et al. 1992, ApJ, 392, 497 Audit, E., \& Hennebelle, P. 2010, A\&A, 511, A76

Ballesteros-Paredes, J., Vazquez-Semadeni, E., \& Scalo, J. 1999, ApJ, 512, 286

Ballesteros-Paredes, J., Klessen, R., Vazquez-Semadeni, E., \& Scalo, J. 2003, ApJ, 592, 188

Banerjee, R., \& Pudritz, R. E. 2007, ApJ, 660, 470

Banerjee, R., Vazquez-Semadeni, E., Hennebelle P., \& Klessen, R. S. 2009, MNRAS, 398, 1082

Bate, M. 2009, MNRAS, 392, 1363

Batrla, W., \& Menten, K. M. 1988, ApJ, 329, L117

Bieging, J. H., Wilson, T. L., \& Downes, D. 1982, A\&AS, 49, 607

Bontemps, S., Motte, F., Csengeri, T., \& Schneider, N. 2010, A\&A, in press [arXiv:0909.2315]

Bonnell, I., \& Bate, M. 2006, MNRAS, 370, 488

Brunt, C. M. 2003, ApJ, 583, 280

Chandler, C. J., Gear, W. K., \& Chini, R. 1993, MNRAS, 260, 337

Csengeri, T., Bontemps, S., Schneider, N., et al. 2010, submitted [arXiv: 1009:0598]

Davis, C. J., Kumar, M. S. N., Sandell, G., et al. 2007, MNRAS, 374, 29

Dickel, H. R., \& Wendker, H. J. 1978, A\&A, 66, 289

Dickel, J. R., Dickel, H. R., \& Wilson, W. J. 1978, ApJ, 223, 840

Downes, D., \& Rinehart, R. 1966, ApJ, 144, 937

Federrath, C., Klessen, R., \& Schmidt, W. 2008, ApJ, 688, L79

Federrath, C., Roman-Duval, J., Klessen, R., Schmidt, W., \& Mac Low, M.-M. 2010, A\&A, 512, A81

Folini, D., \& Walder, R. 2006, A\&A, 459, 1

Fromang, S., Hennebelle, P., \& Teyssier, R. 2006, A\&A, 457, 371

Garden, R. P., Geballe, T. R., Gatley, I., et al. 1991a, ApJ, 366, 474

Garden, R. P., Hayashi, M., Gatley, I., et al. 1991b, ApJ, 374, 540

Garden, R. P., \& Carlstrom, J. E. 1992, ApJ, 392, 602

Genzel, R., \& Downes, D. 1977, A\&AS, 30, 145

Goldreich, P, \& Kwan, J. 1974, ApJ, 189, 441

Gomez, G. C., Vazquez-Semadeni, E., Shadmehri, M., \& Ballesteros-Paredes, J. 2007, ApJ, 669, 1042

Goodman, A. A., Benson, P. J., Fuller, G. A., \& Myers, P. C. 1993, ApJ, 406, 528

Harris, S. 1973, MNRAS, 162, 5P

Heitsch, F., \& Hartmann, L. W. 2008, ApJ, 689, 290 


\section{N. Schneider et al.: DR21 filament}

Heitsch, F., Hartmann, L. W., Slyz, A. D., et al. 2008, ApJ, 674, 316

Hennebelle, P., \& Audit, E. 2007, A\&A, 465, 431

Hennebelle, P., \& Teyssier, R. 2008, A\&A, 477, 25

Hennebelle, P., Banerjee, R., Vazquez-Semadeni, E., Klessen, R., \& Audit, E. 2008, A\&A, 486, L43

Hennebelle, P., \& Chabrier, G. 2008, ApJ, 684, 395

Hora, J., Bontemps, Megeath, T., et al. 2009, A\&AS, 41, 498

Jakob, H., Kramer, C., Simon, R., et al. 2007, A\&A, 461, 999

Kirby, L. 2009, ApJ, 694, 1056

Klessen, R. S. 2001, ApJ, 550, L77

Klessen, R. S., \& Hennebelle, P. 2010, A\&A, 520, A17

Klessen, R. S., Burkert, A., \& Bate, M. R. 1998, ApJ, 501, 205

Klessen, R. S., Heitsch, F., \& Mac Low, M.-M. 2000, ApJ, 535, 887

Klessen, R. S., Ballesteros-Paredes, J., Vazquez-Semadeni, E., et al. 2005, ApJ, 620, 786

Knödlseder, J. 2000, A\&A, 360, 539

Kumar, M. S. N., Davis, C. J., Grave, J. M. C., et al. 2007, MNRAS, 374, 54

Krumholz, M. 2006, ApJ, 641, L45

Krumholz, M., McKee, C. F., \& Klein, R. I. 2005, ApJ, 618, L33

Lee, J. E., Evans, N. J., \& Shirley, Y. L. 2003, ApJ, 583, 789

Lucas, R., \& Liszt, H. 1998, A\&A, 337, 246

Mac Low, M.-M., \& Klessen, R. 2004, Rev. Modern Phys., 76, 125

Mangum, J. G., Wooten, A., \& Mundy, L. G. 1991, ApJ, 378, 576

Mangum, J. G., Wooten, A., \& Mundy, L. G. 1992, ApJ, 388, 467

Marseille, M., Bontemps, S., van der Tak, F., Herpin, F., \& Purcell, C. R. 2008, A\&A, 488, 579

Marston, A. P., Reach, W. T., Noriega-Crispo, A., et al. 2004, ApJS, 154, 333

Mauersberger, R., Henkel, C., Wilson, T. L., \& Walmsley, C. M. 1986, A\&A, 162,199

McKee, C., \& Tan, J. 2003, ApJ, 585, 850

McKee, C. F., \& Ostriker, E. C. 2007, ARA\&A, 45, 565

Motte, F., Schilke P., \& Lis, D. 2003, ApJ, 582, 277

Motte, F., Bontemps, S., Schilke P., Schneider, N., \& Menten, K. 2007, A\&A, 476,1243
Myers, P. C. 2009, ApJ, 700, 1609

Myers, P. C., Mardones, M., Tafalla, M., et al. 1996, ApJ, 465, L133

Norris, R. P., Booth, R. S., Diamond, P. J., \& Porter, N. D. 1982, MNRAS, 201, 191

Nyman, L. A. 1983, 120, 307

Ossenkopf, V., Trojan, C., \& Stutzki, J. 2001, A\&A, 378, 608

Padoan, P., \& Nordlund, A. 2002, ApJ, 576, 870

Peretto, N., André, P., \& Belloche, A. 2006, A\&A, 445, 979

Peretto, N., Hennebelle, P., \& André, P. 2007, A\&A, 464, 983

Peters, T., Banerjee, R., Klessen, R., et al. 2010, ApJ, 711, 1017

Plambeck, R. L., \& Menten, K. 1990, ApJ, 364, 555

Reipurth, B., \& Schneider, N. 2008, Handbook of star forming regions (ASP)

Richardson, K. J., Sandell, G., Cunningham, C. T., et al. 1994, A\&A, 286, 555

Russell, A. P. G., Bally, J., Padman, R., \& Hills, R. E. 1992, ApJ, 387, 219

Schneider, N., Bontemps, S., Simon, R., et al. 2006, A\&A, 458, 855

Schneider, N., Simon, R., Bontemps, S., Comerón, F., \& Motte, F. 2007, A\&A, 474,873

Schneider, N., Bontemps, S., Simon, R., et al. 2010, A\&A, submitted [arXiv: 1001.2453]

Shepherd, D. S., Kurtz, S. E., \& Testi, L. 2004, ApJ, 601, 952

Tafalla, M., Myers, P. C., Caselli, P., Walmsley, C. M., \& Comito, C. 2002, ApJ, 569,815

Teyssier, R. 2002, A\&A, 385, 337

Vallée, J. P., \& Fiege, J. D. 2006, ApJ, 636, 332

Vazquez-Semadeni, E., Shadmehri, M., \& Ballesteros-Paredes, J. 2002, unpublished [arXiv:0208245]

Vazquez-Semadeni, E., Gomez, G. C., Jappsen, A. K., et al. 2007, ApJ, 657, 870

Vazquez-Semadeni, E., Gonzales, R. F., Ballesteros-Paredes, J., et al. 2008, MNRAS, 390, 769

Vazquez-Semadeni, E., Gomez, G. C., Jappsen, A. K., et al. 2009, ApJ, 707, 1023

Wilson, T. L., \& Mauersberger, R. 1990, A\&A, 239, 305

Wienen, M. 2008, Diplomarbeit, University of Bonn

Yorke, H. W., \& Sonnhalter, C. 2002, ApJ, 569, 846 